\title{
Lattice Boltzmann method for conjugate heat and mass transfer with interfacial jump conditions
}

\author{
Kaikai Guo ${ }^{1}$, Like $\mathrm{Li}^{2}$, Gang Xiao ${ }^{1}$, Nick AuYeung ${ }^{2}$, Renwei Mei ${ }^{2, *}$ \\ ${ }^{1}$ State Key Laboratory of Clean Energy Utilization \\ Zhejiang University, Hangzhou, 310027, China \\ ${ }^{2}$ Department of Mechanical and Aerospace Engineering \\ University of Florida, Gainesville, Florida 32611-6250, USA \\ * Corresponding author \\ Tel.: +1 352392 0888; fax: +1 3523921071 \\ E-mail addresses: kaikai_guo@zju.edu.cn, likelichina@ufl.edu, xiaogangtianmen@zju.edu.cn, \\ nauyeung@ufl.edu, rwmei@ufl.edu
}




\title{
Lattice Boltzmann method for conjugate heat and mass transfer with interfacial jump conditions
}

\author{
Kaikai Guo ${ }^{1}$, Like $\mathrm{Li}^{2}$, Gang Xiao', Nick AuYeung ${ }^{2}$, Renwei Mei ${ }^{2, *}$ \\ ${ }^{1}$ State Key Laboratory of Clean Energy Utilization \\ Zhejiang University, Hangzhou, 310027, China \\ ${ }^{2}$ Department of Mechanical and Aerospace Engineering \\ University of Florida, Gainesville, Florida 32611-6250, USA \\ * Corresponding author \\ Tel.: +1 352392 0888; fax: +1 3523921071 \\ E-mail addresses: kaikai_guo@zju.edu.cn, likelichina@ufl.edu,xiaogangtianmen@zju.edu.cn, \\ nauyeung@ufl.edu,rwmei@ufl.edu
}

\begin{abstract}
In this work we propose an interface treatment for conjugate heat and mass transfer with discontinuities or jumps of temperature (concentration) and/or heat (mass) flux at the interface using the lattice Boltzmann equation (LBE) method. The present interface treatment is based on the second-order accurate boundary condition treatments for Dirichlet and Neumann problems (Li et al. 2013, J. Comput. Phys. 237, 366-395) and second-order accurate interface treatment for standard conjugate heat and mass transfer with the continuity of temperature (concentration) and flux at the interface ( $\mathrm{Li}$ et al. 2014, Phys. Rev. E 89, 043308). The interfacial jump conditions are intrinsically satisfied in the present treatment without iterative computations that are typically needed in conventional finite-difference or finite-volume methods. The interfacial temperature (concentration) values and the fluxes into the adjacent domains are conveniently obtained from the microscopic distribution functions in the LBE model without finite-difference approximations. Since the local intersection link fraction is included in the present treatment,
\end{abstract}


the interfacial geometry is preserved and the present interface schemes are capable of handling curved interfaces. The numerical accuracy and convergence of the present interface schemes are verified with several numerical tests, including (i) one-dimensional (1D) steady diffusion within a two-solid slab; the slab is either aligned with the lattice velocity vector or with an inclination angle, (ii) 2D steady diffusion in a circular domain of two concentric solids, (iii) 3D steady diffusion in a spherical domain of two concentric solids, and (iv) 2D steady convection-diffusion in a channel. The two adjacent domains have different thermal (mass) transport properties and specific temperature (concentration) and flux jump conditions are imposed at the interface in each of those tests. It is verified that the present interface treatment for jump conditions does not introduce additional errors compared to the case without jumps; and second-order accuracy in space is obtained for the interior temperature (concentration) field, the interfacial temperature (concentration) values and interfacial fluxes for straight interfaces aligned with the lattice velocity vector in both diffusion and convection-diffusion problems. The effects of inclined and curved interfacial geometry on the order-of-accuracy of the LBE results are also investigated and the results are compared with previous findings.

Keywords: Lattice Boltzmann equation; conjugate heat and mass transfer; temperature (concentration) jump condition; flux jump condition; order of accuracy

\section{Introduction}

Transport phenomena between different phases or different materials with interfacial discontinuities are often encountered in many science and engineering problems in fluid dynamics, heat and mass transfer, materials science, and chemical reactions. The solutions to 
these interface problems are usually non-smooth or even with discontinuities or jumps across the interface, which poses great challenges to any numerical method applied to solve the interface problems. Development of accurate and efficient numerical schemes to treat the boundary or interfacial jump conditions has attracted much attention in the literature, such as the immersed boundary method (IBM) [1, 2], the immersed interface method (IIM) [3, 4], the ghost fluid method (GFM) [5, 6], the sharp interface Cartesian grid method [7, 8], and the matched interface and boundary (MIB) method [9]. Most of these methods are formulated in the finite difference or finite volume frameworks.

Modeling of heat and mass transfer with the lattice Boltzmann equation (LBE) method has been successfully carried out over the last two decades [10-32]. With the intrinsic features of explicit algorithms, easy implementation and compatibility with parallelization, the LBE method has become an effective alternative numerical method for thermal and mass transport phenomena. The standard LBE method deals with the evolution of the microscopic distribution functions defined in the lattice nodes in the Cartesian coordinates. The macroscopic governing equations for the transport processes can be recovered from the evolution equation through a multiscale analysis, such as the Chapman-Enskog expansion $[14,17]$ or the asymptotic analysis [21].

One crucial component in the LBE model development and implementation is the boundary condition treatment which converts the macroscopic boundary conditions (e.g., Dirichlet boundary values or Neumann flux conditions) into a consistent treatment for the microscopic distribution functions [see Ref. 27 and references therein]. The Dirichlet and 
Neumann boundary condition treatments proposed in [27] preserve the local geometry and have been verified to be second-order accurate in space. Consistent analytical schemes for computing boundary fluxes and boundary temperatures (concentrations) have also been proposed in [27] for Dirichlet and Neumann problems, respectively. On this basis, Ref. [30] also proposed an interface treatment for standard conjugate heat and mass transfer in the LBE method by treating the interface as a shared boundary of zero-thickness for the two adjacent domains.

In this work, we extend the work in [30] and propose a consistent interface treatment for conjugate heat and mass transfer with interfacial temperature (concentration) jump and/or interfacial flux jump conditions. The interfacial jump conditions are intrinsically satisfied in each time step without iterations in analogy with the satisfaction of interfacial continuity for standard conjugate problems without jumps in [30]. The double values for interfacial temperature (concentration) and/or fluxes at the interface are obtained from the microscopic distribution functions in each of the adjacent domains without finite-difference computations. Detailed error assessments for the computed interior and interfacial scalar values and the interfacial fluxes for various types of jump conditions are conducted in three numerical tests for which exact solutions are available. The interfacial geometry in these tests involves straight, inclined, and curved interfaces. The results are compared with those without jumps.

The rest of the paper is organized as follows. Section 2 presents the governing equations and the conjugate jump conditions for interfacial heat and mass transfer problems. The LBE model applied is briefly described in Section 3. The details for the proposed interface treatment 
for jump conditions are given in Section 4. Discussion of numerical results is given in Section 5. And Section 6 concludes the paper.

\section{Heat and mass transfer with interfacial jump conditions}

\subsection{Governing equations}

The governing equation for conjugate heat and mass transfer between Domains 1 and 2 connected by an interface (Fig. 1) is written as a convection-diffusion equation (CDE)

$$
\frac{\partial \phi}{\partial t}+\frac{\partial}{\partial x_{j}}\left(v_{j} \phi\right)=\frac{\partial}{\partial x_{i}}\left(D_{i j} \frac{\partial \phi}{\partial x_{j}}\right)+G,
$$

where $\phi$ is a scalar variable denoting the temperature in heat transfer or concentration in mass transfer problems, $t$ is the time, $v_{j}$ is the velocity component in the $x_{j}$ direction, $D_{i j}$ is the diffusion coefficient tensor, and $G$ is the general source term.

\subsection{Interfacial conjugate conditions with jumps}

We consider the general case at the interface with a temperature (concentration) jump $\phi_{\text {jump }}$

$$
\Phi_{d f}=\Phi_{d s}+\phi_{\text {jump }}
$$

where $\Phi_{d f}$ and $\Phi_{d s}$ are the interfacial temperature (concentration) values for the two domains, and a heat (mass) flux jump $q_{\text {jump }}^{\mathrm{HT}}\left(q_{\text {jump }}^{\mathrm{MT}}\right)$

$$
-k_{f} \frac{\partial \phi_{f}}{\partial n_{f}}=k_{s} \frac{\partial \phi_{s}}{\partial n_{s}}+q_{\mathrm{jump}}^{\mathrm{HT}}, \text { for heat transfer, }
$$

or

$$
-D_{f} \frac{\partial \phi_{f}}{\partial n_{f}}=D_{s} \frac{\partial \phi_{s}}{\partial n_{s}}+q_{\mathrm{jump}}^{\mathrm{MT}}, \text { for mass transfer, }
$$


where $k_{f, s}$ are the thermal conductivities and $D_{f, s}$ are the thermal or mass transfer diffusivities of the materials in the two domains, respectively.

The flux jump conditions in Eqs. (3a, 3b) can be written in a general form as

$$
\Phi_{n f}=-D_{f} \frac{\partial \phi_{f}}{\partial n_{f}}=\sigma D_{s} \frac{\partial \phi_{s}}{\partial n_{s}}+q_{\text {jump }}=-\sigma \Phi_{n s}+q_{\text {jump }}
$$

where $\Phi_{n f}$ and $\Phi_{n s}$ are the interfacial fluxes in the normal directions; $\sigma=1, q_{\text {jump }}=q_{\text {jump }}^{\mathrm{MT}}$ in

mass transfer, and $\sigma=\left(\rho c_{p}\right)_{s} /\left(\rho c_{p}\right)_{f}, q_{\text {jump }}=q_{\text {jump }}^{\mathrm{HT}} /\left(\rho c_{p}\right)_{f}$ in heat transfer problems with $\rho$ being the density and $c_{p}$ the heat capacity.

It is noted that when $\phi_{\text {jump }}=0$ and $q_{\text {jump }}=0$, Eqs. (2) and (4) reduce to the standard conjugate heat (mass) transfer problem with the continuity of temperature (concentration) and fluxes at the interface [30].

\section{Lattice Boltzmann models for the convection-diffusion equation}

There have been various LBE models [16, 18-26] proposed for solving Eq. (1). The multiple-relaxation-time (MRT) D3Q7 and D2Q5 (DnQm denotes $m$ discrete lattice velocities in $n$ dimensions) models proposed by Yoshida and Nagaoka [21] are used in the present work for their simplicity, second-order accuracy in space and first-order accuracy in time, and the capability of simulating anisotropic diffusion problems.

With the collision operator presented in the moment space, the lattice Boltzmann equation for the evolution of the microscopic distribution function, $g(\mathbf{x}, \xi, t)$, is written as [21, 27]

$$
g_{\alpha}\left(\mathbf{x}+\mathbf{e}_{\alpha} \delta t, t+\delta t\right)-g_{\alpha}(\mathbf{x}, t)=-\left[\mathbf{M}^{-1} \mathbf{S}\left(\mathbf{m}(\mathbf{x}, t)-\mathbf{m}^{(\mathrm{eq})}(\mathbf{x}, t)\right)\right]_{\alpha}+\omega_{\alpha} G(\mathbf{x}, t) \delta t,
$$


where $g_{\alpha}(\mathbf{x}, t) \equiv g\left(\mathbf{x}, \xi_{\alpha}, t\right), \mathbf{x}$ is the spatial vector, $\xi$ is the particle velocity vector in the phase space $(\mathbf{x}, \boldsymbol{\xi})$ and it is discretized to a small set of discrete velocities $\left\{\boldsymbol{\xi}_{\alpha} \mid \alpha=0,1, \ldots, m-1\right\}$, $\mathbf{e}_{\alpha}$ is the $\alpha$ th discrete velocity vector $\left(\left\{\mathbf{e}_{\alpha}\right\}=(0,0,0),( \pm 1,0,0),(0, \pm 1,0)\right.$, and $(0,0, \pm 1)$ for D3Q7, and $\left\{\mathbf{e}_{\alpha}\right\}=(0,0),( \pm 1,0)$, and $(0, \pm 1)$ for D2Q5), $\mathbf{M}$ is a matrix to transform the distribution functions $\mathbf{g}$ to their moments $\mathbf{m}$ by $\mathbf{m}=\mathbf{M g}$, the equilibrium moments of the distribution functions are explicitly defined as $\mathbf{m}^{(\mathrm{eq})}=(0, u \phi, v \phi, w \phi, a \phi, 0,0)^{\mathrm{T}}$ with $u, v$, and $w$ being the macroscopic velocity components in the Cartesian coordinates and $a$ being a constant related to the weight coefficients $\omega_{\alpha}$ [27], $\mathbf{S}$ is a matrix of relaxation coefficients $\tau_{i j}$, and $\delta t$ is the time step.

The asymptotic analysis in [21] showed that when the relaxation coefficients, $\tau_{i j}$, in the relaxation matrix $\mathbf{S}$ are related to the diffusion coefficients, $D_{i j}$, as

$$
\tau_{i j}=\frac{1}{2} \delta_{i j}+\frac{\delta t}{\varepsilon_{D}(\delta x)^{2}} D_{i j}
$$

the leading-order solution of the $\mathrm{CDE}$ (1) is obtained from the moment of the distribution functions

$$
\phi(\mathbf{x}, t)=\sum_{\alpha=0}^{m-1} g_{\alpha}(\mathbf{x}, t),
$$

with second-order accuracy in space and first-order accuracy in time. In Eq. (6), $\delta_{i j}$ is the Kronecker delta and $\varepsilon_{D}$ is a constant related to the weight coefficients $\omega_{\alpha}$.

For efficient computations, the evolution equation (5) is usually solved in two steps collision step:

$$
\hat{g}_{\alpha}(\mathbf{x}, t)=g_{\alpha}(\mathbf{x}, t)-\left[\mathbf{M}^{-1} \mathbf{S}\left(\mathbf{m}(\mathbf{x}, t)-\mathbf{m}^{(\mathrm{eq})}(\mathbf{x}, t)\right)\right]_{\alpha}+\omega_{\alpha} G(\mathbf{x}, t) \delta t, \text { and }
$$

streaming step: 


$$
g_{\alpha}\left(\mathbf{x}+\mathbf{e}_{\alpha} \delta t, t+\delta t\right)=\hat{g}_{\alpha}(\mathbf{x}, t)
$$

where $\hat{g}_{\alpha}$ represents the post-collision state. It is noted that an efficient implementation of the collision step does not require the storage of $\hat{g}_{\alpha}$. The collision step in Eq. (8) is completely local and the streaming step in Eq. (9) is simple and requires little computational effort. The specific matrices $\mathbf{M}$ and $\mathbf{S}$ and the coefficients $\omega_{\alpha}$ for the D3Q7 and D2Q5 LBE models, and the details about the model implementations can be found in [21, 27].

\section{Interface treatment in LBE for jump conditions}

In order to convert the macroscopic conjugate conditions into those for the microscopic distribution functions, Li et al. [30] proposed a second-order accurate interface treatment for the standard conjugate conditions without jumps based on the boundary condition treatments proposed in [27] for Dirichlet and Neumann problems. The same methodology can be extended to develop appropriate interface treatment for conjugate conditions with jumps.

Following [30], as one considers the interface as a shared boundary of zero-thickness by the two domains, the relationships between the microscopic distribution functions and the macroscopic interfacial $\phi$ values and their fluxes hold for each of the two domains. From the

Dirichlet condition treatment, the following can be obtained

$$
\left\{\begin{array}{l}
g_{\bar{\alpha}}\left(\mathbf{x}_{f}, t+\delta t\right)=c_{d 1} \hat{g}_{\alpha}\left(\mathbf{x}_{f}, t\right)+c_{d 2} \hat{g}_{\alpha}\left(\mathbf{x}_{f f}, t\right)+c_{d 3} \hat{g}_{\bar{\alpha}}\left(\mathbf{x}_{f}, t\right)+c_{d 4} \varepsilon_{D} \Phi_{d f}, \text { in Domain } 1, \\
g_{\alpha}\left(\mathbf{x}_{s}, t+\delta t\right)=c_{d 1}^{*} \hat{g}_{\bar{\alpha}}\left(\mathbf{x}_{s}, t\right)+c_{d 2}^{*} \hat{g}_{\bar{\alpha}}\left(\mathbf{x}_{s s}, t\right)+c_{d 3}^{*} \hat{g}_{\alpha}\left(\mathbf{x}_{s}, t\right)+c_{d 4}^{*} \varepsilon_{D} \Phi_{d s}, \text { in Domain } 2 .
\end{array}\right.
$$

Similarly, from the Neumann condition treatment, we have

$$
\left\{\begin{array}{l}
g_{\bar{\alpha}}\left(\mathbf{x}_{f}, t+\delta t\right)=c_{n 1} \hat{g}_{\alpha}\left(\mathbf{x}_{f}, t\right)+c_{n 2} \hat{g}_{\alpha}\left(\mathbf{x}_{f f}, t\right)+c_{n 3} \hat{g}_{\bar{\alpha}}\left(\mathbf{x}_{f}, t\right)+c_{n 4}(\delta t / \delta x) \Phi_{n \bar{\alpha}}, \text { in Domain 1, } \\
g_{\alpha}\left(\mathbf{x}_{s}, t+\delta t\right)=c_{n 1}^{*} \hat{g}_{\bar{\alpha}}\left(\mathbf{x}_{s}, t\right)+c_{n 2}^{*} \hat{g}_{\bar{\alpha}}\left(\mathbf{x}_{s s}, t\right)+c_{n 3}^{*} \hat{g}_{\alpha}\left(\mathbf{x}_{s}, t\right)+c_{n 4}^{*}(\delta t / \delta x) \Phi_{n \alpha}, \text { in Domain 2, }
\end{array}\right.
$$


where $\Phi_{n \bar{\alpha}}$ and $\Phi_{n \alpha}$ are the respective interfacial fluxes along the discrete lattice velocity directions $\mathbf{e}_{\bar{\alpha}}$ and $\mathbf{e}_{\alpha}, \mathbf{x}_{f}$ and $\mathbf{x}_{f f}$ are the first and second interior lattice nodes along $\mathbf{e}_{\bar{\alpha}}$ direction in Domain $1\left(\mathbf{x}_{f f}=\mathbf{x}_{f}+\mathbf{e}_{\alpha} \delta t\right)$, and $\mathbf{x}_{s}$ and $\mathbf{x}_{s s}$ are the lattice nodes along $\mathbf{e}_{\alpha}$ direction in Domain $2\left(\mathbf{x}_{s s}=\mathbf{x}_{s}+\mathbf{e}_{\alpha} \delta t=\mathbf{x}_{s}-\mathbf{e}_{\bar{\alpha}} \delta t\right)$, respectively. All the coefficients in Eqs. $(10,11)$ are only related to the local geometry at the interface. Specifically, the "one-parameter" family for the coefficients $c_{d i}(i=1,2,3$ and 4$)$ in the Dirichlet condition treatment is [27]

$$
c_{d 2}=-\frac{2 \Delta c_{d 1}+1}{2 \Delta+1}, c_{d 3}=\frac{c_{d 1}+2 \Delta}{2 \Delta+1}, \text { and } c_{d 4}=\frac{-c_{d 1}+1}{2 \Delta+1} .
$$

where $c_{d 1}$ is an adjust parameter, and the link fraction $\Delta$ is defined as $\Delta=\left\|\mathbf{x}_{f}-\mathbf{x}_{w}\right\| /\left\|\mathbf{x}_{f}-\mathbf{x}_{s}\right\|\left(\mathbf{x}_{w}\right.$ is the interface node, see Fig. 1); the coefficients $c_{n i}(i=1,2,3$ and 4) in the Neumann condition treatment are [27]

$$
c_{n 1}=1, \quad c_{n 2}=-\frac{2 \Delta-1}{2 \Delta+1}, c_{n 3}=\frac{2 \Delta-1}{2 \Delta+1}, \text { and } c_{n 4}=\frac{2}{2 \Delta+1} ;
$$

and the coefficients $c_{d i}^{*}$ and $c_{n i}^{*}\left(i=1,2,3\right.$ and 4) are related to $c_{d i}$ and $c_{n i}$ as $c_{d i}^{*}=c_{d i}\left(\Delta^{*}\right)=c_{d i}(1-\Delta)$, and $c_{n i}^{*}=c_{n i}\left(\Delta^{*}\right)=c_{n i}(1-\Delta)$ since $\Delta^{*}=\left\|\mathbf{x}_{s}-\mathbf{x}_{w}\right\| /\left\|\mathbf{x}_{f}-\mathbf{x}_{s}\right\|=1-\Delta$ [30].

When $\mathbf{e}_{\bar{\alpha}}$ is aligned with the interface normal directions, $\Phi_{n \bar{\alpha}}=\Phi_{n f}$ and $\Phi_{n \alpha}=\Phi_{n s}$ are readily noticed; thus Eqs. (2, 4, 10a, 10b, 11a, and 11b) consist a linear system of six equations. The six unknowns $g_{\bar{\alpha}}\left(\mathbf{x}_{f}, t+\delta t\right), g_{\alpha}\left(\mathbf{x}_{s}, t+\delta t\right), \Phi_{d f}, \Phi_{d s}, \Phi_{n f}$ and $\Phi_{n s}$, can be analytically solved (see Section 4.2 below). For general cases, however, one needs to first obtain $\Phi_{n \bar{\alpha}}$ and $\Phi_{n \alpha}$ from the normal fluxes $\Phi_{n f}$ and $\Phi_{n s}$. The Cartesian decomposition method [27] for this conversion is used, which gives 


$$
\begin{aligned}
\Phi_{n \bar{\alpha}}= & \left\{\frac{1}{c_{d 4}^{\prime}}\left[\left(c_{n 1}^{\prime}-c_{d 1}^{\prime}\right) \hat{g}_{\beta}\left(\mathbf{x}_{f}^{\prime}, t\right)+\left(c_{n 2}^{\prime}-c_{d 2}^{\prime}\right) \hat{g}_{\beta}\left(\mathbf{x}_{f f}^{\prime}, t\right)+\left(c_{n 3}^{\prime}-c_{d 3}^{\prime}\right) \hat{g}_{\bar{\beta}}\left(\mathbf{x}_{f}^{\prime}, t\right)\right] \sin \theta\right. \\
& -\frac{1}{c_{d 4}}\left[\left(c_{n 1}-c_{d 1}\right) \hat{g}_{\alpha}\left(\mathbf{x}_{f}, t\right)+\left(c_{n 2}-c_{d 2}\right) \hat{g}_{\alpha}\left(\mathbf{x}_{f f}, t\right)+\left(c_{n 3}-c_{d 3}\right) \hat{g}_{\bar{\alpha}}\left(\mathbf{x}_{f}, t\right)\right] \sin \theta \\
& \left.+\frac{c_{n 4}^{\prime}}{c_{d 4}^{\prime}} \frac{\delta t}{\delta x} \Phi_{n f}\right\} /\left[\frac{c_{n 4}}{c_{d 4}} \frac{\delta t}{\delta x} \sin \theta+\frac{c_{n 4}^{\prime}}{c_{d 4}^{\prime}} \frac{\delta t}{\delta x} \cos \theta\right], \\
\Phi_{n \alpha}= & \left\{\frac{1}{c_{d 4}^{\prime}}\left[\left(c_{n 1}^{\prime}-c_{d 1}^{\prime}\right) \hat{g}_{\bar{\beta}}\left(\mathbf{x}_{s}^{\prime}, t\right)+\left(c_{n 2}^{\prime}-c_{d 2}^{\prime}\right) \hat{g}_{\bar{\beta}}\left(\mathbf{x}_{s s}^{\prime}, t\right)+\left(c_{n 3}^{\prime}-c_{d 3}^{\prime}\right) \hat{g}_{\beta}\left(\mathbf{x}_{s}^{\prime}, t\right)\right] \sin \theta\right. \\
& -\frac{1}{c_{d 4}^{*}}\left[\left(c_{n 1}^{*}-c_{d 1}^{*}\right) \hat{g}_{\alpha}\left(\mathbf{x}_{s}, t\right)+\left(c_{n 2}^{*}-c_{d 2}^{*}\right) \hat{g}_{\alpha}\left(\mathbf{x}_{s s}, t\right)+\left(c_{n 3}^{*}-c_{d 3}^{*}\right) \hat{g}_{\bar{\alpha}}\left(\mathbf{x}_{s}, t\right)\right] \sin \theta \\
& \left.+\frac{c_{n 4}^{\prime}}{c_{d 4}^{\prime}} \frac{\delta t}{\delta x} \Phi_{n s}\right\} /\left[\frac{c_{n 4}^{*}}{c_{d 4}^{*}} \frac{\delta t}{\delta x} \sin \theta+\frac{c_{n 4}^{\prime}}{c_{d 4}^{\prime}} \frac{\delta t}{\delta x} \cos \theta\right],
\end{aligned}
$$

where $\theta$ is the angle between lattice vector $\mathbf{e}_{\bar{\alpha}}$ and the normal $\mathbf{n}$ at the interface node $\mathbf{x}_{w}, \hat{g}_{\bar{\beta}}$ and $\hat{g}_{\beta}$ are the post-collision distribution functions along $\mathbf{e}_{\bar{\beta}}$ and $\mathbf{e}_{\beta}$ that are perpendicular to $\mathbf{e}_{\bar{\alpha}}$ and $\mathbf{e}_{\alpha}$, and they are computed at the intersection nodes $\mathbf{x}_{f}^{\prime}=\mathbf{x}_{s}^{\prime}=\mathbf{x}_{w}, \mathbf{x}_{f f}^{\prime}=\mathbf{x}_{w}+\mathbf{e}_{\bar{\beta}} \delta t$, and $\mathbf{x}_{s s}^{\prime}=\mathbf{x}_{w}+\mathbf{e}_{\beta} \delta t$ (see Fig. 1) with a three-point interpolation or extrapolation of the neighboring distribution functions [30]. The coefficients $c_{d i}^{\prime}$ and $c_{n i}^{\prime}(i=1,2,3$ and 4) are determined from Eqs. (12) and (13) by setting $\Delta=0$, i.e., $c_{d i}^{\prime}=c_{d i}\left(\Delta^{\prime}\right)=c_{d i}(\Delta=0)$, and $c_{n i}^{\prime}=c_{n i}\left(\Delta^{\prime}\right)=c_{n i}(\Delta=0)$ [30].

\subsection{General conjugate interface treatment}

Following [30], the general interface treatment for conjugate heat and mass transfer with jump conditions can be expressed as

$$
\begin{aligned}
g_{\bar{\alpha}}\left(\mathbf{x}_{f}, t+\delta t\right) & =A_{1}^{f} \hat{g}_{\alpha}\left(\mathbf{x}_{f}, t\right)+A_{2}^{f} \hat{g}_{\alpha}\left(\mathbf{x}_{f f}, t\right)+A_{3}^{f} \hat{g}_{\bar{\alpha}}\left(\mathbf{x}_{f}, t\right) \\
& +B_{1}^{f} \hat{g}_{\bar{\alpha}}\left(\mathbf{x}_{s}, t\right)+B_{2}^{f} \hat{g}_{\bar{\alpha}}\left(\mathbf{x}_{s s}, t\right)+B_{3}^{f} \hat{g}_{\alpha}\left(\mathbf{x}_{s}, t\right) \\
& +C_{1}^{f} \hat{g}_{\beta}\left(\mathbf{x}_{f}^{\prime}, t\right)+C_{2}^{f} \hat{g}_{\beta}\left(\mathbf{x}_{f f}^{\prime}, t\right)+C_{3}^{f} \hat{g}_{\bar{\beta}}\left(\mathbf{x}_{f}^{\prime}, t\right) \\
& +\sigma C_{1}^{f} \hat{g}_{\bar{\beta}}\left(\mathbf{x}_{s}^{\prime}, t\right)+\sigma C_{2}^{f} \hat{g}_{\bar{\beta}}\left(\mathbf{x}_{s s}^{\prime}, t\right)+\sigma C_{3}^{f} \hat{g}_{\beta}\left(\mathbf{x}_{s}^{\prime}, t\right) \\
& +\chi_{q} q_{\text {jump }}+\chi_{t} \phi_{\text {jump }},
\end{aligned}
$$




$$
\begin{aligned}
g_{\alpha}\left(\mathbf{x}_{s}, t+\delta t\right) & =A_{1}^{s} \hat{g}_{\bar{\alpha}}\left(\mathbf{x}_{s}, t\right)+A_{2}^{s} \hat{g}_{\bar{\alpha}}\left(\mathbf{x}_{s s}, t\right)+A_{3}^{s} \hat{g}_{\alpha}\left(\mathbf{x}_{s}, t\right) \\
& +B_{1}^{s} \hat{g}_{\alpha}\left(\mathbf{x}_{f}, t\right)+B_{2}^{s} \hat{g}_{\alpha}\left(\mathbf{x}_{f f}, t\right)+B_{3}^{s} \hat{g}_{\bar{\alpha}}\left(\mathbf{x}_{f}, t\right) \\
& +\sigma C_{1}^{s} \hat{g}_{\bar{\beta}}\left(\mathbf{x}_{s}^{\prime}, t\right)+\sigma C_{2}^{s} \hat{g}_{\bar{\beta}}\left(\mathbf{x}_{s s}^{\prime}, t\right)+\sigma C_{3}^{s} \hat{g}_{\beta}\left(\mathbf{x}_{s}^{\prime}, t\right) \\
& +C_{1}^{s} \hat{g}_{\beta}\left(\mathbf{x}_{f}^{\prime}, t\right)+C_{2}^{s} \hat{g}_{\beta}\left(\mathbf{x}_{f f}^{\prime}, t\right)+C_{3}^{s} \hat{g}_{\bar{\beta}}\left(\mathbf{x}_{f}^{\prime}, t\right) \\
& +\gamma_{q} q_{\text {jump }}+\gamma_{t} \phi_{\text {jump }},
\end{aligned}
$$

where the coefficients $A_{i}^{f}, B_{i}^{f}, C_{i}^{f}, A_{i}^{s}, B_{i}^{s}$, and $C_{i}^{s},(i=1,2,3)$ for the post-collision distribution functions are the same as those given in [30], and the coefficients $\chi_{q}, \chi_{t}, \gamma_{q}$, and $\gamma_{t}$ can be similarly determined when one substitutes Eqs. (14a, b) into Eqs. (11a, b) and solves the linear system of equations $(2,4,10 \mathrm{a}, 10 \mathrm{~b}, 11 \mathrm{a}$, and 11b). For completeness, the coefficients are listed below

$$
\left\{\begin{array}{l}
A_{i}^{f}=\left[\frac{(\sigma+1) c_{d 4}^{\prime} c_{d i}}{c_{d 4} c_{d 4}^{*} c_{n 4}^{\prime}} \sin \theta+\left(\frac{\sigma c_{d i}}{c_{d 4} c_{n 4}^{*}}+\frac{c_{n i}}{c_{d 4}^{*} c_{n 4}}\right) \cos \theta\right] / P \\
B_{i}^{f}=\sigma\left(\frac{c_{n i}^{*}-c_{d i}^{*}}{c_{d 4}^{*} c_{n 4}^{*}}\right) \cos \theta / P \\
C_{i}^{f}=\left(\frac{c_{n i}^{\prime}-c_{d i}^{\prime}}{c_{d 4}^{*} c_{n 4}^{\prime}}\right) \sin \theta / P \\
\chi_{q}=\frac{1}{c_{d 4}^{*} P} \\
\chi_{t}=\varepsilon_{D} \sigma\left[\frac{c_{d 4}^{\prime}}{c_{d 4}^{*} c_{n 4}^{\prime}} \sin \theta+\frac{1}{c_{n 4}^{*}} \cos \theta\right] / P
\end{array},(i=1,2,3),\right.
$$

and 


$$
\left\{\begin{array}{l}
A_{i}^{s}=\left[\frac{(1+\sigma) c_{d 4}^{\prime} c_{d i}^{*}}{c_{d 4}^{*} c_{d 4} c_{n 4}^{\prime}} \sin \theta+\left(\frac{c_{d i}^{*}}{c_{d 4}^{*} c_{n 4}}+\frac{\sigma c_{n i}^{*}}{c_{d 4} c_{n 4}^{*}}\right) \cos \theta\right] / P \\
B_{i}^{s}=\left(\frac{c_{n i}-c_{d i}}{c_{d 4} c_{n 4}}\right) \cos \theta / P \\
C_{i}^{s}=\left(\frac{c_{n i}^{\prime}-c_{d i}^{\prime}}{c_{d 4} c_{n 4}^{\prime}}\right) \sin \theta / P \\
\gamma_{q}=\frac{1}{c_{d 4} P} \\
\gamma_{t}=-\varepsilon_{D}\left[\frac{c_{d 4}^{\prime}}{c_{d 4} c_{n 4}^{\prime}} \sin \theta+\frac{1}{c_{n 4}} \cos \theta\right] / P
\end{array},(i=1,2,3) .\right.
$$

with

$$
P=\frac{(\sigma+1) c_{d 4}^{\prime}}{c_{d 4} c_{d 4}^{*} c_{n 4}^{\prime}} \sin \theta+\left(\frac{\sigma}{c_{d 4} c_{n 4}^{*}}+\frac{1}{c_{d 4}^{*} c_{n 4}}\right) \cos \theta .
$$

As stressed in [30], with $g_{\alpha}\left(\mathbf{x}_{f}, t+\delta t\right)$ and $g_{\bar{\alpha}}\left(\mathbf{x}_{s}, t+\delta t\right)$ obtained from the present conjugate interface treatment, the macroscopic interfacial temperature (concentration) and interfacial fluxes in the lattice velocity directions $\mathbf{e}_{\bar{\alpha}}$ and $\mathbf{e}_{\alpha}$ can be directly obtained from the microscopic distribution functions without finite-difference calculations. Those analytical schemes are

$$
\begin{aligned}
& \Phi_{d f}=\frac{1}{c_{d 4} \varepsilon_{D}}\left[g_{\bar{\alpha}}\left(\mathbf{x}_{f}, t+\delta t\right)-c_{d 1} \hat{g}_{\alpha}\left(\mathbf{x}_{f}, t\right)-c_{d 2} \hat{g}_{\alpha}\left(\mathbf{x}_{f f}, t\right)-c_{d 3} \hat{g}_{\bar{\alpha}}\left(\mathbf{x}_{f}, t\right)\right], \\
& \Phi_{d s}=\frac{1}{c_{d 4}^{*} \varepsilon_{D}}\left[g_{\alpha}\left(\mathbf{x}_{s}, t+\delta t\right)-c_{d 1}^{*} \hat{g}_{\bar{\alpha}}\left(\mathbf{x}_{s}, t\right)-c_{d 2}^{*} \hat{g}_{\bar{\alpha}}\left(\mathbf{x}_{s s}, t\right)-c_{d 3}^{*} \hat{g}_{\alpha}\left(\mathbf{x}_{s}, t\right)\right] \\
& \Phi_{n \bar{\alpha}}=\frac{\delta x}{c_{n 4} \delta t}\left[g_{\bar{\alpha}}\left(\mathbf{x}_{f}, t+\delta t\right)-c_{n 1} \hat{g}_{\alpha}\left(\mathbf{x}_{f}, t\right)-c_{n 2} \hat{g}_{\alpha}\left(\mathbf{x}_{f f}, t\right)-c_{n 3} \hat{g}_{\bar{\alpha}}\left(\mathbf{x}_{f}, t\right)\right], \text { and } \\
& \Phi_{n \alpha}=\frac{\delta x}{c_{n 4}^{*} \delta t}\left[g_{\alpha}\left(\mathbf{x}_{s}, t+\delta t\right)-c_{n 1}^{*} \hat{g}_{\bar{\alpha}}\left(\mathbf{x}_{s}, t\right)-c_{n 2}^{*} \hat{g}_{\bar{\alpha}}\left(\mathbf{x}_{s s}, t\right)-c_{n 3}^{*} \hat{g}_{\alpha}\left(\mathbf{x}_{s}, t\right)\right]
\end{aligned}
$$


It is emphasized that the interface jumps $\phi_{\text {jump }}$ and $q_{\text {jump }}$ are naturally incorporated into the interface treatment in Eqs. (15a) and (15b), and they are not explicitly shown in Eqs. $(18,19)$.

For special situations such as straight interface cases and those with zero-tangential flux along the interface, the conjugate interface treatment can be greatly simplified by decoupling the interfacial fluxes in the lattice velocity directions in each domain [30]. The decoupled interface treatment for jump conditions is thus presented in the next.

\subsection{Decoupled conjugate interface treatment}

For straight interfaces whose normal directions are aligned with the lattice vectors $\mathbf{e}_{\bar{\alpha}}$ and $\mathbf{e}_{\alpha}$, the following relationships are noticed

$$
\Phi_{n \bar{\alpha}}=\Phi_{n f} \text { and } \Phi_{n \alpha}=\Phi_{n s} \quad \text { when } \mathbf{e}_{\bar{\alpha}} / / \mathbf{n}
$$

In addition, if the tangential flux $\Phi_{t}$ along an interface is known to be zero the following simple projection rule holds (see Fig. 1)

$$
\Phi_{n \bar{\alpha}}=\Phi_{n f} \cos \theta, \text { and } \Phi_{n \alpha}=\Phi_{n s} \cos \theta \quad \text { when } \Phi_{t}=0
$$

In these two special cases, the coupling of the interfacial fluxes in $\mathbf{e}_{\bar{\alpha}}$ and $\mathbf{e}_{\bar{\beta}}$ directions disappears [30] and the conjugate interface treatment in Eqs. (15a) and (15b) is thus simplified to

$$
\begin{aligned}
g_{\bar{\alpha}}\left(\mathbf{x}_{f}, t+\delta t\right) & =A_{1}^{f} \hat{g}_{\alpha}\left(\mathbf{x}_{f}, t\right)+A_{2}^{f} \hat{g}_{\alpha}\left(\mathbf{x}_{f f}, t\right)+A_{3}^{f} \hat{g}_{\bar{\alpha}}\left(\mathbf{x}_{f}, t\right) \\
& +B_{1}^{f} \hat{g}_{\bar{\alpha}}\left(\mathbf{x}_{s}, t\right)+B_{2}^{f} \hat{g}_{\bar{\alpha}}\left(\mathbf{x}_{s s}, t\right)+B_{3}^{f} \hat{g}_{\alpha}\left(\mathbf{x}_{s}, t\right), \\
& +\chi_{q} q_{\text {jump }}+\chi_{t} \phi_{\text {jump }}, \\
g_{\alpha}\left(\mathbf{x}_{s}, t+\delta t\right) & =A_{1}^{s} \hat{g}_{\bar{\alpha}}\left(\mathbf{x}_{s}, t\right)+A_{2}^{s} \hat{g}_{\bar{\alpha}}\left(\mathbf{x}_{s s}, t\right)+A_{3}^{s} \hat{g}_{\alpha}\left(\mathbf{x}_{s}, t\right) \\
& +B_{1}^{s} \hat{g}_{\alpha}\left(\mathbf{x}_{f}, t\right)+B_{2}^{s} \hat{g}_{\alpha}\left(\mathbf{x}_{f f}, t\right)+B_{3}^{s} \hat{g}_{\bar{\alpha}}\left(\mathbf{x}_{f}, t\right), \\
& +\gamma_{q} q_{\text {jump }}+\gamma_{t} \phi_{\text {jump }},
\end{aligned}
$$

with

$$
A_{i}^{f}=\left(\frac{\sigma c_{d i}}{c_{d 4} c_{n 4}^{*}}+\frac{c_{n i}}{c_{d 4}^{*} c_{n 4}}\right) / P, \quad B_{i}^{f}=\sigma\left(\frac{c_{n i}^{*}-c_{d i}^{*}}{c_{d 4}^{*} c_{n 4}^{*}}\right) / P,(i=1,2,3),
$$




$$
\begin{aligned}
& A_{i}^{s}=\left(\frac{c_{d i}^{*}}{c_{d 4}^{*} c_{n 4}}+\frac{\sigma c_{n i}^{*}}{c_{d 4} c_{n 4}^{*}}\right) / P, \quad B_{i}^{s}=\left(\frac{c_{n i}-c_{d i}}{c_{d 4} c_{n 4}}\right) / P,(i=1,2,3), \\
& \chi_{q}=\frac{1}{c_{d 4}^{*} P}, \quad \chi_{t}=\frac{\varepsilon_{D} \sigma}{c_{n 4}^{*} P}, \quad \gamma_{q}=\frac{1}{c_{d 4} P}, \quad \gamma_{t}=-\frac{\varepsilon_{D}}{c_{n 4} P}
\end{aligned}
$$

and

$$
P=\frac{\sigma}{c_{d 4} c_{n 4}^{*}}+\frac{1}{c_{d 4}^{*} c_{n 4}}
$$

\subsection{Particular conjugate interface schemes for jump conditions}

Three particular boundary schemes for the Dirichlet condition were presented in [26] with different choices of the adjustable coefficient $c_{d 1}$ as a function of the local $\Delta$ value. Their numerical stability and second-order accuracy were verified in [27]. Correspondingly, three particular conjugate interface schemes were provided in [30] for no jump conditions. In this work, three interface schemes for jump conditions are also presented using those choices of $c_{d 1}$ and maintaining the relationships for the other coefficients for second-order accuracy. For 2-D problems, the coefficients for $A_{i}^{f}, B_{i}^{f}, C_{i}^{f}, A_{i}^{s}, B_{i}^{s}$, and $C_{i}^{s},(i=1,2,3)$ are the same as those in Table 1 in [30], and the coefficients for $\chi_{q}, \chi_{t}, \gamma_{q}$, and $\gamma_{t}$ are listed in Table 1 of the present paper.

For the decoupled interface treatment in Eqs. (22a) and (22b), the coefficients in Eqs. (23a), (23b), (24) and (25) are greatly simplified with a direct substitution of $\sin \theta=0$ and $\cos \theta=$ 1 into the coefficients in Eqs. (16a, 16b, 17) [30]. Those coefficients are listed separately in Table 2. 
When the interfaces are straight and located "halfway" between the lattice nodes $(\Delta=$ $0.5)$, the substitution of $\Delta=0.5$ in Table 2 gives the further simplified decoupled conjugate treatment for jump conditions

$$
\begin{aligned}
& g_{\bar{\alpha}}\left(\mathbf{x}_{f}, t+\delta t\right)=\left(\frac{1-\sigma}{1+\sigma}\right) \hat{g}_{\alpha}\left(\mathbf{x}_{f}, t\right)+\left(\frac{2 \sigma}{1+\sigma}\right) \hat{g}_{\bar{\alpha}}\left(\mathbf{x}_{s}, t\right)+\left(\frac{1}{1+\sigma}\right) q_{\mathrm{jump}}+\left(\frac{\varepsilon_{D} \sigma}{1+\sigma}\right) \phi_{\mathrm{jump}} \\
& g_{\alpha}\left(\mathbf{x}_{s}, t+\delta t\right)=-\left(\frac{1-\sigma}{1+\sigma}\right) \hat{g}_{\bar{\alpha}}\left(\mathbf{x}_{s}, t\right)+\left(\frac{2}{1+\sigma}\right) \hat{g}_{\alpha}\left(\mathbf{x}_{f}, t\right)+\left(\frac{1}{1+\sigma}\right) q_{\mathrm{jump}}-\left(\frac{\varepsilon_{D}}{1+\sigma}\right) \phi_{\mathrm{jump}} .
\end{aligned}
$$

It is noted that only the local distributions at $\mathbf{x}_{f}$ and $\mathbf{x}_{s}$ are required in this "half lattice division" scheme. Furthermore, for the most simplified case of $\Delta=0.5$ and $\sigma=1$, Eqs. (26a-b) reduces to

$$
\begin{aligned}
& g_{\bar{\alpha}}\left(\mathbf{x}_{f}, t+\delta t\right)=\hat{g}_{\bar{\alpha}}\left(\mathbf{x}_{s}, t\right)+q_{\text {jump }} / 2+\varepsilon_{D} \phi_{\text {jump }} / 2, \\
& g_{\alpha}\left(\mathbf{x}_{s}, t+\delta t\right)=\hat{g}_{\alpha}\left(\mathbf{x}_{f}, t\right)+q_{\text {jump }} / 2-\varepsilon_{D} \phi_{\text {jump }} / 2 .
\end{aligned}
$$

\section{Results and discussion}

To study the numerical accuracy of the proposed interface treatment for conjugate heat and mass transfer with jump conditions, we present results from four numerical tests for which exact solutions are available. The first test is the one-dimensional (1D) steady diffusion with a source term in a slab of two different solid materials. The rectangular two-solid slab is either aligned with the lattice links (Section 5.1) or with an inclination angle (Section 5.2). The second test is for two-dimensional (2D) steady diffusion within a circular domain. The third test deals with three-dimensional (3D) steady diffusion within a spherical domain. The two materials on each side of the curved interfaces in Tests (ii) and (iii) also have different thermal or mass transport properties. The fourth test studies the effect of convection in a confined 2D channel. For all tests, discontinuous interfacial flux and/or temperature (concentration) jump conditions 
are applied. The numerical accuracy of the LBE solutions for the interior distribution of $\phi$, the interfacial $\phi$ values and the interfacial fluxes are systematically investigated.

\subsection{One-D steady diffusion in a horizontal slab with sources}

The configuration of the slab and the square lattice distribution are schematically depicted in Fig. 2, in which the interface is fixed at $x=L / 2$. The two solids have different transport properties characterized by the thermal conductivity ratio $k_{1} / k_{2}$. One should also note that for steady heat conduction problems the ratio of the diffusion coefficients in the LBE model is set to the thermal conductivity ratio, i.e., $D_{1} / D_{2}=k_{1} / k_{2}$ so that $\sigma=\left(\rho c_{p}\right)_{1} /\left(\rho c_{p}\right)_{2}=1$ in Eq. (4). In the 2D computational domain, periodic boundary conditions are applied on the top and bottom walls; simple Dirichlet boundary conditions, $\phi(x=0)=\phi(x=L)=0$, are imposed on the two ends; and four different conjugate conditions at the interface: (i) standard conjugate conditions with continuous temperature and flux ("no jump"), (ii) with continuous temperature and a flux jump (“flux jump”), (iii) with continuous flux and a temperature jump (“temp jump”), and (iv) with both flux and temperature jumps ("flux \& temp jumps"), are separately examined.

To obtain non-trivial solutions, a source term, $G_{i}(x)=D_{i} S(x)(i=1,2)$, is included in each domain. The governing $\mathrm{CDE}$ is thus written as

$$
\begin{aligned}
& D_{1} \frac{\partial^{2} \phi_{1}}{\partial x^{2}}+D_{1} S(x)=0, \quad 0 \leq x \leq L / 2, \\
& D_{2} \frac{\partial^{2} \phi_{2}}{\partial x^{2}}+D_{2} S(x)=0, \quad L / 2 \leq x \leq L,
\end{aligned}
$$

with

$$
S(x)=\frac{12 a}{L^{2}}\left(\frac{x}{L}\right)^{2}+\frac{6 b}{L^{2}}\left(\frac{x}{L}\right)+\frac{2 c}{L^{2}}, \quad 0 \leq x \leq L,
$$


where $a, b$, and $c$ are constant values. In this study, three different types of heat source distributions, including constant $(a=0, b=0, c=1)$, linear $(a=0, b=1, c=0)$ and quadratic ( $a$ $=1, b=0, c=0$ ) distributions, are examined to assess the impact of the interfacial jump conditions on the accuracy of the LBE solutions.

The general conjugate conditions with both jumps at the interface are

$$
\begin{aligned}
& \phi_{1}=\phi_{2}+\phi_{\text {jump }} \text { at } x=L / 2, \text { and } \\
& -D_{1} \frac{\partial \phi_{1}}{\partial x}=-D_{2} \frac{\partial \phi_{2}}{\partial x}+q_{\text {jump }} \text { at } x=L / 2 .
\end{aligned}
$$

Note that Eqs. (30a) and (30b) reduce to the flux jump condition when $\phi_{\text {jump }}=0, q_{\text {jump }} \neq 0$, to the temperature jump condition when $\phi_{\text {jump }} \neq 0, q_{\text {jump }}=0$, and to the no jump condition when $\phi_{\text {jump }}=$ $0, q_{\mathrm{jump}}=0$. Recalling the boundary conditions at the two ends, the expressions for the exact solutions are

$$
\begin{aligned}
& \phi_{1, \mathrm{ex}}=-\left[a(x / L)^{4}+b(x / L)^{3}+c(x / L)^{2}\right]+\lambda_{1} x, \\
& \phi_{2, \mathrm{ex}}=-\left[a(x / L)^{4}+b(x / L)^{3}+c(x / L)^{2}\right]+\lambda_{2} x+\lambda_{3},
\end{aligned}
$$

where

$$
\begin{aligned}
& \lambda_{1}=\frac{D_{1}(2 a+3 b+4 c)+D_{2}(6 a+5 b+4 c)-4 L q_{\text {jump }}+8 D_{2} \phi_{\text {jump }}}{4 L\left(D_{1}+D_{2}\right)}, \\
& \lambda_{2}=\frac{D_{1}(6 a+5 b+4 c)+D_{2}(2 a+3 b+4 c)+4 L q_{\text {jump }}+8 D_{1} \phi_{\text {jump }}}{4 L\left(D_{1}+D_{2}\right)}, \text { and } \\
& \lambda_{3}=\frac{\left(D_{2}-D_{1}\right)(2 a+b)-4 L q_{\text {jump }}-8 D_{1} \phi_{\text {jump }}}{4\left(D_{1}+D_{2}\right)} .
\end{aligned}
$$

The interfacial temperatures and fluxes for the two subdomains are thus

$$
\begin{aligned}
& \phi_{1, \text { ex_int }}=-(a / 16+b / 8+c / 4)+L \lambda_{1} / 2, \text { and } \\
& \phi_{2, \text { ex_int }}=-(a / 16+b / 8+c / 4)+L \lambda_{2} / 2+\lambda_{3} ;
\end{aligned}
$$




$$
\begin{aligned}
& q_{1, \text { ex_int }}=-\left.D_{1} \frac{\partial \phi_{1}}{\partial x}\right|_{L / 2}=D_{1}\left(\frac{a}{2 L}+\frac{3 b}{4 L}+\frac{c}{L}\right)-D_{1} \lambda_{1}, \text { and } \\
& q_{2, \text { ex_int }}=-\left.D_{2} \frac{\partial \phi_{2}}{\partial x}\right|_{L / 2}=D_{2}\left(\frac{a}{2 L}+\frac{3 b}{4 L}+\frac{c}{L}\right)-D_{2} \lambda_{2} .
\end{aligned}
$$

The placement of the slab in the square lattice is illustrated in Fig. 2. For each $\Delta$ value shown in Fig. 2, the decoupled interface treatment in Eqs. (22a, b) with the coefficients determined in Table 2 for the three particular schemes is applied. The corresponding Schemes 1-3 with the same choices of the adjustable coefficient $c_{d 1}$ are used for the Dirichlet boundary conditions at the two ends. The implementation of the periodical boundary conditions in the $y$-direction follows that in [27]. For all the results shown below, six lattice nodes are used in the $y$-direction and the LBE solutions along the $y$-direction are exactly the same.

To assess the accuracy of the interface schemes we define the absolute error

$$
E_{\mathrm{abs}}=\phi_{1,2 \_\mathrm{LBE}}-\phi_{1,2 \_\mathrm{ex}},
$$

and the relative $L_{2}$-norm errors

$$
\begin{aligned}
& E_{2}=\left[\sum_{x, y}\left(\phi_{\mathrm{LBE}}-\phi_{\mathrm{ex}}\right)^{2} / \sum_{x, y} \phi_{\mathrm{ex}}^{2}\right]^{1 / 2}, \\
& E_{2 \_ \text {tint_1,2 }}=\left[\sum_{x=L / 2, y}\left(\phi_{1,2 \_\mathrm{LBE}}-\phi_{1,2 \_\mathrm{ex}}\right)^{2} / \sum_{x=L / 2, y} \phi_{1,2 \_\mathrm{ex}}^{2}\right]^{1 / 2}, \\
& E_{2 \_\mathrm{qint} \_1,2}=\left[\sum_{x=L / 2, y}\left(\left.D_{1,2} \frac{\partial \phi_{1,2}}{\partial x}\right|_{\mathrm{LBE}}-\left.D_{1,2} \frac{\partial \phi_{1,2}}{\partial x}\right|_{\mathrm{ex}}\right)^{2} / \sum_{x=L / 2, y}\left(\left.D_{1,2} \frac{\partial \phi_{1,2}}{\partial x}\right|_{\mathrm{ex}}\right)^{2}\right]^{1 / 2},
\end{aligned}
$$

where $E_{2}$ contains the relative errors for all the interior lattice nodes in Domains 1 and 2; $E_{2 \_ \text {tint_1, }}$ $E_{2 \_ \text {tint_2 }}$ and $E_{2 \_ \text {qint_1 }}, E_{2 \_ \text {qint_2 }}$ evaluate the relative errors of the interfacial temperatures and fluxes in Domains 1 and 2, respectively. 
For illustration purposes, Fig. 3 (a) shows the profiles of $\phi$ in the $x$-direction for the various jump conditions with a quadratic source distribution. The parameters used are $\tau_{1}=0.75$, $\tau_{2}=0.525$ (thus $\left.D_{1} / D_{2}=10\right), L=42$ and $\Delta=0.25$. The jump conditions $\left(\phi_{\text {jump }}=0.5\right.$ and $q_{\text {jump }}=$ $\left.-D_{1} / L\right)$ at the interface $x=L / 2$ are seen in Fig. 3 (a). The slope change at the interface for the no jump condition is due to the different materials $\left(D_{1} / D_{2}=10\right)$. For all cases, the LBE results agree very well with exact solutions. The comparison is further examined by the absolute errors $E_{\text {abs }}$ defined in Eq. (35). The results in Fig. 3 (b) clearly show that for each of the three types of heat source distributions, the four different interfacial conjugate conditions give almost the same absolute errors. It is thus verified that the present interface treatment for various jump conditions on straight interfaces is very accurate, and it does not introduce any additional errors compared with the standard treatment for no jump or continuous conjugate conditions. The numerical results also confirm that the temperature and flux jumps are inherently preserved in the present interface treatment. This test indicates that the LBE method is a very attractive numerical method for conjugate heat and mass transfer problems involving interfacial jumps in the physical variable and/or its normal derivatives.

The behavior of the errors in Fig. 3 (b) for different heat sources can also be understood from a finite-difference (FD) point of view. He et al. [33] shown that when the BGK scheme is applied to solve the pressure-driven fully-developed steady-state channel flow problem, it reduces to a central difference scheme in the lateral direction for the streamwise velocity. We observed this to be the case when the thermal LBE scheme is applied to solve simple diffusion 
problems such as thermal conduction with a continuous source. The FD form of Eq. (28) using central difference scheme can be written as

$$
\frac{\phi_{i+1}-2 \phi_{i}+\phi_{i-1}}{(\delta x)^{2}}+S_{i}=0
$$

After performing Taylor series expansions one obtains

$$
\left.\frac{d^{2} \phi}{d x^{2}}\right|_{i}+S_{i}+\text { T.E. }=0 \text { with T.E. }\left.\sim \frac{1}{12} \frac{d^{4} \phi}{d x^{4}}\right|_{i}(\delta x)^{2},
$$

where T.E. denotes the truncation error that is proportional to $\left.\frac{d^{4} \phi}{d x^{4}}\right|_{i}$. The FD solution can be expressed as $\phi_{\mathrm{FD}}=\phi_{\mathrm{ex}}+E_{\mathrm{abs}}$ with $\left.\frac{d^{2} \phi_{\mathrm{ex}}}{d x^{2}}\right|_{i}+S_{i} \equiv 0$. Substitution of these into Eq. (40) yields

$$
\left.\frac{d^{2} E_{\text {abs }}}{d x^{2}}\right|_{i}+\text { T.E. }=0, \text { or }\left.\frac{d^{2} E_{\text {abs }}}{d x^{2}}\right|_{i}=- \text { T.E. } \sim-\left.\frac{1}{12} \frac{d^{4} \phi}{d x^{4}}\right|_{i}(\delta x)^{2},
$$

Recalling the fourth-order polynomial for $\phi_{1,2}$ in Eqs. $(31 \mathrm{a}, \mathrm{b})$, for the respective constant and linear sources $S=2 / L^{2}$ and $S=\left(6 / L^{2}\right)(x / L)$, we have $\frac{d^{4} \phi}{d x^{4}}=0$, thus $\left.\frac{d^{2} E_{\text {abs }}}{d x^{2}}\right|_{i}=0$ or $E_{\mathrm{abs}}=c_{0}+c_{1} x$, where $c_{0}$ and $c_{1}$ are constants; for the quadratic source $S=\left(12 / L^{2}\right)(x / L)^{2}$ we have $\frac{d^{4} \phi}{d x^{4}}=$ const, thus $\left.\frac{d^{2} E_{\mathrm{abs}}}{d x^{2}}\right|_{i}=c$ or $E_{\mathrm{abs}}=c_{0}^{\prime}+c_{1}^{\prime} x+c x^{2} / 2$, where $c, c_{0}^{\prime}$ and $c_{1}^{\prime}$ are also constants. Therefore the profiles for the absolute errors in Fig. 3 (b) are linear segments for the cases with constant and linear sources and quadratic segments for the case with a quadratic source. The discontinuity in the error profiles for each case is due to the material property difference $\left(D_{1} / D_{2}=10\right)$ and the placement of the interface at $\Delta=0.25$.

To verify the order-of-accuracy of the LBE solutions, Figs. 4-6 show the relative $L_{2}$-norm errors, $E_{2}, E_{2 \_ \text {tint }}$ and $E_{2 \_ \text {qint, }}$ defined in Eqs. (36-38) for the interior temperature field, the interfacial temperatures and fluxes, respectively, versus the grid resolution, $1 / L$, at different $\Delta$ values with all three schemes. For each case the quadratic source $S=\left(12 / L^{2}\right)(x / L)^{2}$ is applied, and both temperature and flux jump conditions with $\phi_{\text {jump }}=0.5$ and $q_{\text {jump }}=-D_{1} / L$ are used. Note that 
the interface is a shared boundary for Domains 1 and 2, thus double values for the interfacial temperature and fluxes are obtained from the two domains as shown in Figs. 5a, 5b, and 6a, 6b, respectively. Clearly, second-order accuracy is obtained for all cases in Figs. 4-6. The variations of $E_{2}, E_{2 \_ \text {tint }}$ and $E_{2 \_ \text {qint }}$ in the whole range of $0 \leq \Delta \leq 1$ are shown in Fig. 7 for $\phi_{\text {jump }}=0.5$ and $q_{\text {jump }}=-D_{1} / L(L=42)$. The $E_{2 \_ \text {tint }}$ and $E_{2 \_ \text {qint }}$ values are obtained from Domain 1 and Scheme 2 is used for the interface and boundary conditions. For each of the three $L_{2}$-norm errors, the minimum error occurs at different $\Delta$ values. The error behavior is consistent with previous findings in $[27,28]$. Similar trends are observed for the $E_{2 \_ \text {tint }}$ and $E_{2 \_ \text {qint }}$ values obtained from Domain 2 and using other interface and boundary schemes; thus they are not shown for brevity.

To compare the effects of the different interface jump conditions, Figs. 8-10 show the respective results of $E_{2}, E_{2 \_ \text {tint }}$ and $E_{2 \_ \text {qint }}$ versus $1 / L$ for the no jump $\left(\phi_{\text {jump }}=0, q_{\text {jump }}=0\right)$, flux jump $\left(\phi_{\text {jump }}=0, q_{\text {jump }}=-D_{1} / L\right)$, and temperature jump $\left(\phi_{\text {jump }}=0.5, q_{\text {jump }}=0\right)$ cases. For each case, the quadratic source is applied and $\Delta=0.5$ is used for the interface as well as the two boundaries at $x=0$ and $x=L$. Again, second-order accuracy is verified for all cases.

The differences in the magnitude of the relative errors for the different jump conditions in Figs. 8-10 are attributed to the fact that the absolute errors for the various jump conditions are almost the same as shown in Fig. 3 (b), while the exact solutions given by Eqs. $(31,33,34)$ for the interior field and the interfacial $\phi$ values and fluxes vary with different jump conditions. For instance, as indicated by Fig. 3 (a), the magnitude of the $\phi$ profiles, $\bar{\phi}_{\mathrm{ex}}=\left(\sum_{x, y} \phi_{\mathrm{ex}}^{2}\right)^{1 / 2}$, for the three different jump conditions follows the order of $\left(\bar{\phi}_{\mathrm{ex}}\right)_{\text {no jump }}>\left(\bar{\phi}_{\mathrm{ex}}\right)_{\text {temp jump }}>\left(\bar{\phi}_{\mathrm{ex}}\right)_{\text {flux jump }}$, thus the corresponding $L_{2}$-norm errors $E_{2}$ in Fig. 8 are $\left(E_{2}\right)_{\text {no jump }}<\left(E_{2}\right)_{\text {temp jump }}<\left(E_{2}\right)_{\text {flux jump }}$; the 
interfacial $\phi$ values is in the order of $\left|\phi_{\text {tint_1 } 1}\right|_{\text {temp jump }}>\left|\phi_{\text {tint }}\right|_{\text {no jump }}>\left|\phi_{\text {tint } \_2}\right|_{\text {temp jump }}=\left|\phi_{\text {tint }}\right|_{\text {flux jump }}$, thus $\left(E_{2 \_ \text {tint_1 } 1}\right)_{\text {temp jump }}<\left(E_{2_{-} \text {tint }}\right)_{\text {no jump }}<\left(E_{2 \_ \text {tint_2 }}\right)_{\text {temp jump }}=\left(E_{2_{-} \text {tint }}\right)_{\text {flux jump }}$ is observed in Fig. 9; and the interfacial fluxes in the order of $\left|\phi_{\text {qint_ } \_1}\right|_{\text {flux jump }}>\left|\phi_{\text {qint_ } 2}\right|_{\text {flux jump }}=\left|\phi_{\text {qint }}\right|_{\text {temp jump }}>\left|\phi_{\text {qint }}\right|_{\text {no jump }}$ result in $\left(E_{2 \_ \text {qint_1 } 1}\right)_{\text {flux jump }}<\left(E_{2_{-} \text {qint_2 } 2}\right)_{\text {flux jump }}=\left(E_{2 \_ \text {qint }}\right)_{\text {temp jump }}<\left(E_{2 \_ \text {qint }}\right)_{\text {no jump }}$ in Fig. 10.

It should also be noted that for the "no jump" and "flux jump" cases, the interfacial temperature is continuous, thus $E_{2 \_ \text {tint_1 }}=E_{2 \_ \text {tint_2 }}$ as given in Fig. 9. For the "temp jump" case, the interfacial temperature is discontinuous, thus different relative errors of $E_{2 \_ \text {tint_1 }}$ and $E_{2 \_ \text {tint_2 }}$ are obtained from the two domains. The analogy for the interfacial fluxes is observed in Fig. 10, i.e., $E_{2 \_ \text {qint_1 }}=E_{2 \_ \text {qint_2 }}$ for the "no jump" and "temp jump" cases; while different $E_{2 \_q i n t \_1}$ and $E_{2 \_ \text {qint_2 }}$ values are obtained for the "flux jump" case from the two domains.

\subsection{One-D steady diffusion in an inclined slab with a heat source}

This test considers the same 1D diffusion problem with a quadratic source $S=$ $\left(12 / L^{2}\right)(x / L)^{2}$ as that in Section 5.1 but the two-solid slab is placed in the square lattice with an inclination angel $\theta$ (see Fig. 11). This test is designed to examine the accuracy of the present interface treatment for jump conditions when the normal direction of the interface is not aligned with the lattice velocity vectors.

The exact solutions for the interior field and the interfacial values are the same as those in Eqs. $(31,33,34)$. The relaxation coefficients are still fixed at $\tau_{1}=0.75$ and $\tau_{2}=0.525$. With the local link fractions prescribed, the Dirichlet boundary treatment is applied for $\phi(x=0)=0$ and $\phi$ $(x=L)=0$ on the two ends; periodical boundary condition is again implemented in the $y$-direction; for the interface at $x=L / 2$, the general interface treatment in Eqs. $(15 \mathrm{a}, 15 \mathrm{~b})$ is 
applied. Although it is known that $\Phi_{t}=0$ along the interface for this problem, Eq, (21) is not used in order to examine the effect of the arbitrary angles between the normal and the lattice vectors on the accuracy of the LBE solutions.

The relative $L_{2}$-norm errors, $E_{2}, E_{2 \_ \text {tint }}$ and $E_{2 \_ \text {qint }}$, defined in Eqs. (36-38), versus $1 / L$ are shown in Figs. 12-14, respectively. The inclination angle $\theta=\pi / 4$ and the lattice link fraction $\Delta=$ 0.4 (see Fig. 11) are used for all cases. The choice of the adjustable coefficient $c_{d 1}$ from Scheme 3 is used for both the Dirichlet boundary conditions and the conjugate conditions at the interface. First-order accuracy is obtained for the three quantities in Figs. 12-14 for all three types of conjugate conditions, including the "no jump" $\left(\phi_{\text {jump }}=0, q_{\text {jump }}=0\right)$, "flux jump" $\left(\phi_{\text {jump }}=0, q_{\text {jump }}\right.$ $\left.=-D_{1} / L\right)$, and "temp jump" $\left(\phi_{\text {jump }}=0.5, q_{\text {jump }}=0\right)$ conditions. The degradation from second-order accuracy in Figs. 8-10 to first-order accuracy in Figs. 12-14 is due to the implementation of the Cartesian decomposition method, Eq. (14). To elucidate the different error behavior in Section 5.1 where the decoupled interface treatment is applied for the interface aligned with the lattice velocity vector and in the present test where the coupled interface treatment is used for the inclined interface, Fig. 15 shows the profiles for the absolute error $E_{\mathrm{abs}}$ defined in Eq. (35) along the inclined $x$-axis for different jump conditions. Again, all three jump conditions lead to the same absolute errors, which is consistent with that in Fig. 3 (b). However, the magnitude of the absolute errors in Fig. 15 is much larger than that in Fig. 3 (b). This naturally leads to a closer examination on the accuracy of the interfacial conditions using the coupled interface treatment proposed in this work. 
The LBE results show that the interfacial $\phi_{\text {int }}$ values at all the interface nodes obtained from the two domains satisfy $\phi_{\text {int } \_1}=\phi_{\text {int } \_2}+\phi_{\text {jump }}$ exactly for both $\phi_{\text {jump }}=0$ and $\phi_{\text {jump }}=0.5$. For the interfacial fluxes, two error measures, err $=\left|-D_{1} \frac{\partial \phi_{1}}{\partial x^{\prime}}\right|_{\mathrm{LBE}}+\left.D_{2} \frac{\partial \phi_{2}}{\partial x^{\prime}}\right|_{\mathrm{LBE}}-q_{\mathrm{jump}} \cos \theta \mid$ and R.E. $=\operatorname{err} /\left|D_{1} \frac{\partial \phi_{1}}{\partial x^{\prime}}\right|_{\mathrm{ex}} \mid$, are defined where $x^{\prime}$ is in the lattice velocity direction (see Fig. 11). The results for err and R.E. versus the grid resolution are plotted in Figs. 16 (a, b), respectively. The identical numerical results for the absolute error, err, in Fig. 16 (a) for all three jump conditions indicate that the present coupled interface treatment for jump conditions does not introduce any extra errors compared with that for the no jump condition. The dominant error is the same for all three jump conditions and it is attributed to the Cartesian decomposition method that satisfies the interfacial flux continuity or the interfacial flux jump condition only to the first-order accuracy as shown in Fig. 16 (b).

The variations of $E_{2}, E_{2 \_ \text {tint }}$ and $E_{2 \_ \text {qint }}$ in the whole range of $0 \leq \Delta \leq 1$ are depicted in Fig. 17 for two combined jumps $\phi_{\text {jump }}=0.5$ and $q_{\text {jump }}=-D_{1} / L$ with $L=41 / \cos (\pi / 4)$. All three $L_{2}$-norm errors show the same pattern and their minimum values occur at the same $\Delta$ value near 0.5 . This is similar to the results shown in Fig. 7; however the magnitude of the errors are much larger with the inclined interface because the order-of-accuracy is lower.

Recalling that the exact solution for the tangential flux $\Phi_{t}$ along the interface is zero for this problem, we also conducted another test by explicitly taking into account this information as in Eq. (21) and using the decoupled interface treatment. As expected, second-order accuracy is

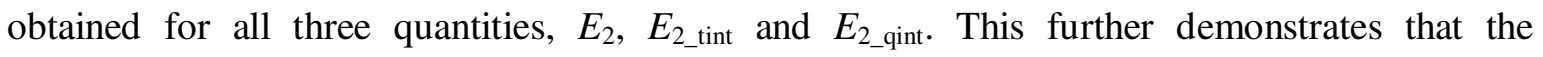


degradation of numerical accuracy from second- to first-order in the coupled interface treatment for conjugate transport problems is attributed to the Cartesian decomposition method. Since $\Phi_{t}$ at the interface is generally not known, such second-order accuracy is not practically meaningful. The results are not shown here for brevity.

\subsection{Two-D steady diffusion in a circular domain with interfacial jump conditions}

To study the applicability and accuracy of the general interface treatment in Eqs. (15a, $15 b)$ for jump conditions on curved geometry, steady diffusion in a circular domain is considered here. The computational domain and the lattice layout are shown in Fig. 18 following the similar work in [30]. The curved geometry at the interface and the outer boundary is preserved by using the actual link fractions $\Delta x$ and $\Delta y$ for the lattice nodes adjacent to the interface and the outer boundary (see $P_{1}$ and $P_{2}$ in Fig. 18). The presently proposed interface treatment at $r=R_{1}$ and the outer boundary treatment at $r=R_{2}$ are implemented.

For the steady diffusion problem with an outer Dirichlet boundary condition

$$
\phi_{2}\left(r=R_{2}\right)=\cos (n \varphi), n=\text { integer, }
$$

and interfacial conjugate conditions with jumps

$$
\begin{aligned}
& \phi_{1}=\phi_{2}+\phi_{\text {jump }}=\phi_{2}+\phi_{0} \cos (n \varphi) \text { at } r=R_{1}, \text { and } \\
& -D_{1} \frac{\partial \phi_{1}}{\partial r}=-D_{2} \frac{\partial \phi_{2}}{\partial r}+q_{\text {jump }}=-D_{2} \frac{\partial \phi_{2}}{\partial r}+q_{0} \cos (n \varphi) \text { at } r=R_{1},
\end{aligned}
$$

the exact solutions for $\phi_{1,2}$ are

$$
\begin{aligned}
& \phi_{1, \mathrm{ex}}(r, \varphi)=a_{1} r^{n} \cos (n \varphi), 0 \leq r \leq R_{1}, \\
& \phi_{2, \mathrm{ex}}(r, \varphi)=\left(a_{2} r^{n}+a_{3} r^{-n}\right) \cos (n \varphi), R_{1} \leq r \leq R_{2},
\end{aligned}
$$

where 


$$
\begin{aligned}
& a_{1}=\frac{2 n R_{1}^{-n-1} R_{2}^{-n} D_{2}-\left(R_{1}^{-2 n}-R_{2}^{-2 n}\right) q_{0}+n R_{1}^{-1}\left(R_{1}^{-2 n}+R_{2}^{-2 n}\right) D_{2} \phi_{0}}{n R_{1}^{n-1}\left(R_{1}^{-2 n}-R_{2}^{-2 n}\right) D_{1}+n R_{1}^{n-1}\left(R_{1}^{-2 n}+R_{2}^{-2 n}\right) D_{2}}, \\
& a_{2}=\frac{n R_{1}^{-n-1} R_{2}^{-n}\left(D_{1}+D_{2}\right)+R_{2}^{-2 n} q_{0}+n R_{1}^{-1} R_{2}^{-2 n} D_{1} \phi_{0}}{n R_{1}^{n-1}\left(R_{1}^{-2 n}-R_{2}^{-2 n}\right) D_{1}+n R_{1}^{n-1}\left(R_{1}^{-2 n}+R_{2}^{-2 n}\right) D_{2}}, \\
& a_{3}=\frac{n R_{1}^{n-1} R_{2}^{-n}\left(D_{2}-D_{1}\right)-q_{0}-n R_{1}^{-1} D_{1} \phi_{0}}{n R_{1}^{n-1}\left(R_{1}^{-2 n}-R_{2}^{-2 n}\right) D_{1}+n R_{1}^{n-1}\left(R_{1}^{-2 n}+R_{2}^{-2 n}\right) D_{2}} .
\end{aligned}
$$

Since the tangential fluxes along the circular interface and outer boundary are not equal to zero, the fluxes in the 2D lattice velocity directions are fully coupled and the general interface treatment in Eqs. $(15 \mathrm{a}, 15 \mathrm{~b})$ is employed for all the results shown below.

Similar to the diffusion problem in the 1D slab in Sections 5.1 and 5.2, the absolute errors of $E_{\mathrm{abs}}=\phi_{\mathrm{LBE}}-\phi_{\mathrm{ex}}$ for the various jump conditions are examined first. Figs. 19 (a-f) compare the $E_{\text {abs }}$ values along the radius at $\varphi=0$ and $\varphi=\pi / 4$ for the "no jump" $\left(\phi_{\text {jump }}=0, q_{\text {jump }}=0\right)$, "flux jump" $\left(\phi_{\text {jump }}=0, q_{\text {jump }}=n D_{2} / R_{1}\right)$, "temp jump" $\left(\phi_{\text {jump }}=0.5, q_{\text {jump }}=0\right)$, and "flux \& temp jumps" $\left(\phi_{\text {jump }}=0.5, q_{\text {jump }}=n D_{2} / R_{1}\right)$ conditions with Scheme 2 used for both the outer boundary and the interface. In Figs. $19(\mathrm{a}, \mathrm{b})$ the same material property is assumed with $\tau_{1}=\tau_{2}=0.75$ and $n=4$ is used in Eq. (42). It is clear that the various jump conditions result in different absolute errors; the "no jump" case has the smallest error magnitude and the case with a "temp jump" has much higher error magnitude than that with a "flux jump" condition. In Figs. 19 (c-f) different material properties are assumed (the relaxation coefficients are $\tau_{1}=0.525, \tau_{2}=0.75$ for $D_{2} / D_{1}=10$, and $\tau_{1}$ $=0.75, \tau_{2}=0.525$ for $\left.D_{2} / D_{1}=0.1\right)$ and $n=1$ is used for all. It is noted in Figs. 19 (c, d) that the "flux jump" case has the largest error magnitude while in Figs. 19 (e, f) the "temp jump" case has the largest. Compared to the results in Figs. 3 (b) and Fig. 15 where almost the same absolute errors are obtained for various jump conditions on a straight interface, the different absolute error 
behavior in the present test is attributed to the curved geometry of the interface. It is also cautioned that the differences in their error magnitude depend on the specific problem and a general conclusion cannot be reached.

To examine the order-of-accuracy of the general interface treatment for conjugate jump conditions on the curved interface, Figs. $20-22$ show the relative $L_{2}$-norm errors $E_{2}, E_{2 \_ \text {tint }}$ and $E_{2 \_ \text {qint }}$ defined in Eqs. (36-38) versus the grid resolution, $1 / R_{1}$. The parameters used are $R_{2} / R_{1}=2$, $n=1, \tau_{1}=0.525$ and $\tau_{2}=0.75$. All three Schemes 1-3 are compared in these plots. For the present test $E_{2}$ includes the relative errors at all the interior lattice nodes within $0 \leq r \leq R_{2} ; E_{2 \_ \text {tint }}$ and $E_{2 \_ \text {qint }}$ are evaluated at all the interface nodes $\left(r=R_{1}\right)$ and the interfacial fluxes are in the lattice velocity directions. Both $E_{2 \_ \text {tint }}$ and $E_{2 \_ \text {qint }}$ are computed within Domain 1 . Their respective results computed within Domain 2 have the same convergence order and are thus not shown. Note that the exact solutions for the interfacial fluxes in the Cartesian lattice directions, such as $\left.\Phi_{n x, 1}\right|_{\mathrm{ex}}=-\left.D_{1} \frac{\partial \phi_{1}}{\partial x}\right|_{\mathrm{ex}}$, are obtained from the projections of the interfacial fluxes in both the normal and tangential directions along the circular interface, e.g., $\left.\Phi_{n x, 1}\right|_{\mathrm{ex}}=\left.\Phi_{n r, 1}\right|_{\mathrm{ex}} \cos \varphi+\left.\Phi_{n t, 1}\right|_{\mathrm{ex}} \sin \varphi=-\left.D_{1} \frac{\partial \phi_{1}}{\partial r}\right|_{r=R_{1}, \mathrm{ex}} \cos \varphi+\left.D_{1} \frac{1}{R_{1}} \frac{\partial \phi_{1}}{\partial \varphi}\right|_{r=R_{1}, \mathrm{ex}} \sin \varphi \quad$ for $0 \leq \varphi \leq$ $\pi / 4$. Very fine mesh is used in the present study to ensure the asymptotic convergence of the LBE results. The highest resolution in [30] is $\varepsilon_{\min }=1 / R_{1}=1 / 64.25=0.0156$; in the present work, $\varepsilon_{\min }$ $=1 / 200.25=0.00499$.

It is observed that all the results for no jump $\left(\phi_{\text {jump }}=0, q_{\text {jump }}=0\right)$, flux jump $\left(\phi_{\text {jump }}=0\right.$, $\left.q_{\text {jump }}=D_{2} / R_{1}\right)$, and temperature jump $\left(\phi_{\text {jump }}=0.5, q_{\text {jump }}=0\right)$ conditions are very similar in terms of the convergence trend. The overall convergence is first-order for each case in Figs. 20-22. The 
"quadratic convergence" as indicted by the line of "slope $=2$ " in [30] is only observed in the range of $\varepsilon=1 / R_{1}>0.0156$. Thus the statement "the computed interior temperature field and the interfacial temperature values are second-order accurate" in [30] for the no jump conjugate conditions is incorrect due to the use of insufficiently large $R_{1}$. The degradation from second-order convergence for the decoupled interface treatment to first-order for the general interface treatment is due to the Cartesian decomposition method for obtaining the interfacial fluxes in the lattice velocity directions from the normal fluxes. As verified in [27] for the diffusion problem in a circular domain with a Neumann boundary condition, second-order accurate interior and boundary temperatures can be obtained when the exact fluxes in the lattice velocity directions are imposed; while only first-order accurate results are obtained when the Cartesian decomposition method is applied and the tangential flux condition is not explicitly prescribed. The diffusion problem in Section 5.2 has also demonstrated that when the Cartesian decomposition method is used only first-order accuracy is obtained for the LBE results of $E_{2}$, $E_{2 \_ \text {tint }}$ and $E_{2 \_ \text {qint }}$ even for conjugate transport on an inclined straight interface. The first-order accurate interfacial fluxes in Fig. 22 is consistent with the first-order interfacial fluxes in [30] and the first-order boundary fluxes in [27-29, 32] for curved boundaries.

With the present interface treatment for 2-D curved geometry, the temperature and flux jump conditions do not affect the convergence orders. They also have first-order accuracy for the interior and interfacial $\phi$ values and the interfacial fluxes compared with the standard conjugate conditions without jumps.

\subsection{Steady diffusion in a spherical domain with interfacial jump conditions}


In this section we consider steady diffusion in a spherical domain consisting of an inner solid and an outer shell. The 2D axisymmetric LBE model [29] is applied. The 2D computational domain and the lattice layout are schematically depicted in Fig. 23. The origin of the sphere is placed halfway in the lattice link to ensure symmetry and the radii are integers with $R_{2}=2 R_{1}$.

A Dirichlet boundary condition is imposed on the outer surface as

$$
\phi_{2}\left(r=R_{2}\right)=\cos \theta .
$$

The conjugate conditions with interfacial jumps are

$$
\begin{aligned}
& \phi_{1}=\phi_{2}+\phi_{\text {jump }}=\phi_{2}+\phi_{0} \cos \theta \text { at } r=R_{1}, \\
& -D_{1} \frac{\partial \phi_{1}}{\partial r}=-D_{2} \frac{\partial \phi_{2}}{\partial r}+q_{\text {jump }}=-D_{2} \frac{\partial \phi_{2}}{\partial r}+q_{0} \cos \theta \text { at } r=R_{1} .
\end{aligned}
$$

The analytical solutions are

$$
\begin{aligned}
& \phi_{1, \mathrm{ex}}(r, \theta)=b_{1} r \cos \theta, 0 \leq r \leq R_{1}, \\
& \phi_{2, \mathrm{ex}}(r, \theta)=\left(b_{2} r+b_{3} r^{-2}\right) \cos \theta, R_{1} \leq r \leq R_{2},
\end{aligned}
$$

where

$$
\begin{aligned}
& b_{1}=\frac{3 R_{1}^{-3} R_{2}^{-1} D_{2}-\left(R_{1}^{-3}-R_{2}^{-3}\right) q_{0}+R_{1}^{-1}\left(2 R_{1}^{-3}+R_{2}^{-3}\right) D_{2} \phi_{0}}{\left(R_{1}^{-3}-R_{2}^{-3}\right) D_{1}+\left(2 R_{1}^{-3}+R_{2}^{-3}\right) D_{2}}, \\
& b_{2}=\frac{R_{1}^{-3} R_{2}^{-1}\left(D_{1}+2 D_{2}\right)+R_{2}^{-3} q_{0}+R_{1}^{-1} R_{2}^{-3} D_{1} \phi_{0}}{\left(R_{1}^{-3}-R_{2}^{-3}\right) D_{1}+\left(2 R_{1}^{-3}+R_{2}^{-3}\right) D_{2}}, \\
& b_{3}=\frac{R_{2}^{-1}\left(D_{2}-D_{1}\right)-q_{0}-R_{1}^{-1} D_{1} \phi_{0}}{\left(R_{1}^{-3}-R_{2}^{-3}\right) D_{1}+\left(2 R_{1}^{-3}+R_{2}^{-3}\right) D_{2}} .
\end{aligned}
$$

Similar to the test in Section 5.3, the curved geometry at the interface and the outer boundary is preserved by calculating the local link fractions. The coefficient for the adjustable coefficient $c_{d 1}$ is chosen from Scheme 3 and the same choices are used for the interface treatment 
and the outer boundary condition treatment. The relaxation coefficients are $\tau_{1}=0.525$ and $\tau_{2}=$ 0.75 thus $D_{2} / D_{1}=10$.

Figures 24-26 show the relative $L_{2}$-norm errors $E_{2}, E_{2 \_ \text {tint }}$ and $E_{2 \_ \text {qint }}$ for the interior temperature field, the interfacial temperatures and fluxes, respectively, versus the grid resolution, $1 / R_{1}$. The computation procedure for $E_{2}, E_{2 \_ \text {tint }}$ and $E_{2 \_ \text {qint }}$ is the same as that in Section 5.3. The results in Figs. 24-26 over a large range of resolution confirm that overall first-order accuracy is obtained for all three quantities of interest for the "no jump" $\left(\phi_{\text {jump }}=0, q_{\text {jump }}=0\right)$, "flux jump" $\left(\phi_{\text {jump }}=0, q_{\text {jump }}=D_{2} / R_{1}\right)$ and "temp jump" $\left(\phi_{\text {jump }}=0.5, q_{\text {jump }}=0\right)$ conditions.

\subsection{Steady convection-diffusion in a channel with interfacial jump conditions}

To investigate the effect of convection on the applicability and accuracy of the present interface treatment for jump conditions, steady convection-diffusion in a $2 \mathrm{D}$ channel is considered. The computational domain and the lattice layout are sketched in Fig. 27, where a plug flow is assumed for the fluid region with $\mathbf{u}\left(u_{x}, u_{y}\right)=(\mathrm{U}, 0)$ and the solid region $\mathbf{u}\left(u_{x}, u_{y}\right)=$ $(0,0)$. The fluid convection is characterized by the Péclet number $\mathrm{Pe}=U(H-h) / D_{2}$.

Dirichlet boundary conditions are specified on the bottom and top walls

$$
\phi_{1}(y=0)=0, \phi_{2}(y=H)=\cos (k x), k=2 \pi / L,
$$

and the scalar and flux jump conditions imposed at the interface are

$$
\begin{aligned}
& \phi_{1}=\phi_{2}+\phi_{\text {jump }}=\phi_{2}+\phi_{0} \cos (k x) \text { at } y=h, \\
& -D_{1} \frac{\partial \phi_{1}}{\partial y}=-D_{2} \frac{\partial \phi_{2}}{\partial y}+q_{\text {jump }}=-D_{2} \frac{\partial \phi_{2}}{\partial y}-q_{0} \cos (k x) \text { at } y=h .
\end{aligned}
$$

Further assuming periodic boundary conditions in the $x$-direction $\phi_{1,2}(x+L)=\phi_{1,2}(x)$, the analytical solutions are 


$$
\begin{aligned}
& \phi_{1, \mathrm{ex}}(x, y)=\operatorname{Re}\left[a_{1} \mathrm{e}^{i k x} \sinh (k y)\right], 0 \leq y \leq h, \\
& \phi_{2, \mathrm{ex}}(x, y)=\operatorname{Re}\left[e^{i k x}\left(a_{2} e^{\lambda y}+a_{3} e^{-\lambda y}\right)\right], \text { with } \lambda=k \sqrt{1+\frac{i U}{D_{2} k}}, h \leq y \leq H,
\end{aligned}
$$

where "Re" denotes the real part of a complex number and the coefficients $a_{1}-a_{3}$ are the solution to the following equations

$$
\left[\begin{array}{ccc}
0 & e^{\lambda H} & e^{-\lambda H} \\
\sinh (k h) & -e^{\lambda h} & -e^{-\lambda h} \\
D_{1} k \cosh (k h) & -D_{2} \lambda e^{\lambda h} & D_{2} \lambda e^{-\lambda h}
\end{array}\right]\left(\begin{array}{l}
a_{1} \\
a_{2} \\
a_{3}
\end{array}\right)=\left(\begin{array}{l}
1 \\
\phi_{0} \\
q_{0}
\end{array}\right) .
$$

The relative $L_{2}$-norm errors $E_{2}, E_{2 \_ \text {tint }}$ and $E_{2 \_ \text {qint }}$ defined in Eqs. (36-38) for the interior $\phi$ field, the interfacial $\phi_{\text {int }}$ and flux values, versus the grid resolution, $1 / H$, are shown in Figs. 28-30, respectively. The specific jump conditions are "no jump" $\left(\phi_{0}=0, q_{0}=0\right)$, "flux jump" $\left(\phi_{0}=0, q_{0}\right.$ $\left.=D_{2} / h\right)$, "temp jump" $\left(\phi_{0}=0.5, q_{0}=0\right)$, and "flux \& temp jumps" $\left(\phi_{0}=0.5, q_{0}=D_{2} / h\right)$. For all cases, the simulation parameters are $\mathrm{Pe}=20, H / h=2, \tau_{1}=0.75, \tau_{2}=0.525\left(\right.$ thus $\left.D_{2} / D_{1}=0.1\right)$, and $\Delta=0.5$ for the horizontal walls and the interface. Second-order accuracy is verified for all cases in Figs. 28-30 and the results are consistent with those in Figs. 8-10 for the 1D diffusion problem. The relative $L_{2}$-norm errors at different Péclet numbers with "flux \& temp jumps" ( $\phi_{0}=$ $\left.0.5, q_{0}=D_{2} / h\right)$ and $H=64 \delta y$ are shown in Fig. 31. It is clear that convection has a strong effect on the magnitude of the relative errors. The present treatment for interface with jumps in physical variables is capable of handling conjugate heat and mass transfer problems with jump conditions and in the presence of significant convection.

Finally, it is worth noting that for convection-diffusion problems with strong convection effect and a higher Mach number, a deviation term proportional to $\frac{\partial^{2} \phi v_{i}}{\partial t \partial x_{i}}$ in the resulting 
macroscopic equation may be significant [26]. The modified MRT model proposed by Huang and $\mathrm{Wu}[26]$ can eliminate this deviation term from the macroscopic equation by modifying the relaxation matrix. The present work focuses on the treatment of the interfacial jumps in the physical variables and its effects on the solution accuracy. The numerical test cases considered in this study involve steady state condition so that the resulting deviation term is zero in all cases.

\section{Conclusions}

A second-order accurate interface treatment for conjugate heat and mass transfer with interfacial jumps is proposed in the lattice Boltzmann equation (LBE) method. The interfacial temperature (concentration) and flux jump conditions are satisfied at each time step in the LBE computation and no iteration is needed. Thus the standard computational procedure and all the inherent benefits of the LBE method are preserved. For straight interfaces that are aligned with the lattice velocity vector, second-order accuracy for the interior temperature (concentration) field and the interfacial values and fluxes is verified with numerical tests for both diffusion and convection-diffusion problems. It is also confirmed that the present interface treatment for jump conditions does not introduce any additional errors compared with the treatment for standard conjugate problems with no jumps and they have the same convergence orders.

The proposed interface treatment is also applicable to conjugate thermal and mass transport on inclined and curved interfaces as it takes into account the local lattice link fractions. Due to the implementation of the Cartesian decomposition method which converts the interfacial normal fluxes into the fluxes in the lattice velocity directions at the interface nodes, the 
temperature (concentration) continuity or jump condition is exactly satisfied while the flux continuity or jump condition is only satisfied with first-order accuracy. Thus overall first-order accuracy is obtained for the interior and interfacial temperature (concentration) and their interfacial fluxes for conjugate heat and mass transfer on inclined and curved interfaces.

\section{Acknowledgements}

This paper was prepared with the support of the U.S. Department of Energy, ARPA-E, under Award No. DEAR0000184.

\section{References}

[1] C.S. Peskin, The immersed boundary method, Acta Numer. 11, 479-517 (2002).

[2] R. Mittal, G. Iaccarino, Immersed boundary methods, Annu. Rev. Fluid Mech. 37, 239-261 (2005).

[3] R.J. LeVeque, Z.L. Li, The immersed interface method for elliptic equations with discontinuous coefficients and singular sources, SIAM J. Numer. Anal. 31, 1019-1044 (1994).

[4] S.Z. Deng, K. Ito, Z.L. Li, Three-dimensional elliptic solvers for interface problems and applications, J. Comput. Phys. 184, 215-243 (2003).

[5] R.P. Fedkiw, T. Aslam, B. Merriman, S. Osher, A non-oscillatory Eulerian approach to interfaces in multimaterial flows (the ghost fluid method), J. Comput. Phys. 152, 457-492 (1999).

[6] X.D. Liu, R.P. Fedkiw, M. Kang, A boundary condition capturing method for Poisson's equation on irregular domains, J. Comput. Phys. 160, 151-178 (2000).

[7] H.S. Udaykumar, R. Mittal, P. Rampunggoon, A. Khanna, A sharp interface Cartesian grid method for simulating flows with complex moving boundaries, J. Comput. Phys. 174, 345-380 (2001).

[8] S. Marella, S. Krishnan, H. Liu, H.S. Udaykumar, Sharp interface Cartesian grid method I: An easily implemented technique for 3D moving boundary computations, J. Comput. Phys. 210, 1-31 (2005). 
[9] Y.C. Zhou, S. Zhao, M. Feig, G.W. Wei, High order matched interface and boundary method for elliptic equations with discontinuous coefficients and singular sources, $J$. Comput. Phys. 213, 1-30 (2006).

[10]F.J. Alexander, S. Chen, J.D. Sterling, Lattice Boltzmann thermohydrodynamics, Phys. Rev. E 47, R2249 (1993).

[11]E.G. Flekky, Lattice Bhatnagar-Gross-Krook models for miscible fluids, Phys. Rev. E 47, 4247-4257 (1993).

[12]Y. Chen, H. Ohashi, M. Akiyama, Thermal lattice Bhatnagar-Gross-Krook model without nonlinear deviations in macrodynamic equations, Phys. Rev. E 50, 2776 (1994).

[13]X. Shan, Simulation of Rayleigh-Benard convection using a lattice Boltzmann method, Phys. Rev. E 55, 2780 (1997).

[14]X. He, S. Chen, G.D. Doolen, A novel thermal model for the lattice Boltzmann method in incompressible limit, J. Comput. Phys. 146, 282-300 (1998).

[15]P. Lallemand, L.-S. Luo, Theory of the lattice Boltzmann method: acoustic and thermal properties in two and three dimensions, Phys. Rev. E, 68, 036706 (2003).

[16]R.G.M. van der Sman, M.H. Ernst, Convection-diffusion lattice Boltzmann scheme for irregular lattices, J. Comput. Phys. 160, 766-782 (2000).

[17]Z. Guo, C. Zheng, B. Shi, Thermal lattice Boltzmann equation for low Mach number flows: decoupling model, Phys. Rev. E 75, 036704 (2007).

[18]B. Servan-Camas, F. T.-C. Tsai, Lattice Boltzmann method with two relaxation times for advection-diffusion equation: Third order analysis and stability analysis, Adv. Water Resour. 31, 1113-1126 (2008).

[19]B.C. Shi, Z. Guo, Lattice Boltzmann model for nonlinear convection-diffusion equations, Phys. Rev. E 79, 016701 (2009).

[20]A. Mezrhab, M.A. Moussaoui, M. Jami, H. Naji, M. Bouzidi, Double MRT thermal lattice Boltzmann method for simulating convective flows, Phys. Lett. A 374, 3499-3507 (2010).

[21]H. Yoshida, M. Nagaoka, Multiple-relaxation-time lattice Boltzmann model for the convection and anisotropic diffusion equation, J. Comput. Phys. 229, 7774-7795 (2010).

[22]I. Ginzburg, D. d'Humières, A. Kuzmin, Optimal stability of advection-diffusion lattice Boltzmann models with two relaxation times for positive/negative equilibrium, J. Stat. Phys. 139, 1090-1143 (2010).

[23]I. Ginzburg, Multiple anisotropic collisions for advection-diffusion Lattice Boltzmann schemes, Adv. Water Resour. 51, 381-404 (2013).

[24]Z. Chai, T.S. Zhao, Lattice Boltzmann model for the convection-diffusion equation, Phys. Rev. E 87, 063309 (2013).

[25]J. Wang, D. Wang, P. Lallemand, L.-S. Luo, Lattice Boltzmann simulations of thermal convective flows in two dimensions, Comput. Mathe. Appli. 65, 262-286 (2013). 
[26]R. Huang, H. Wu, A modified multiple-relaxation-time lattice Boltzmann model for convection-diffusion equation, J. Comput. Phys. 274, 50-63 (2014).

[27]L. Li, R. Mei, J.F. Klausner, Boundary conditions for thermal lattice Boltzmann equation method, J. Comput. Phys. 237, 366-395 (2013).

[28]L. Li, R. Mei, J.F. Klausner, Heat transfer evaluation on curved boundaries in thermal lattice Boltzmann equation method, ASME J. Heat Transfer 136, 012403 (2014).

[29]L. Li, R. Mei, J.F. Klausner, Multiple-relaxation-time lattice Boltzmann model for the axisymmetric convection diffusion equation, Int. J. Heat Mass Transfer 67, 338-351 (2013).

[30]L. Li, C. Chen, R. Mei, J.F. Klausner, Conjugate heat and mass transfer in the lattice Boltzmann equation method, Phys. Rev. E 89, 043308 (2014).

[31]H. Yoshida, T. Kobayashi, H. Hayashi, T. Kinjo, H. Washizu, K. Fukuzawa, Boundary condition at a two-phase interface in the lattice Boltzmann method for the convection-diffusion equation, Phys. Rev. E 90, 013303 (2014).

[32]L. Li, N. AuYeung, R. Mei, J.F. Klausner, Effects of boundary condition discontinuities on the accuracy of lattice Boltzmann method for heat and mass transfer, Phys. Rev. E, under review (2014).

[33]X. He, Q. Zou, L.-S. Luo, M. Dembo, Analytic solutions of simple flows and analysis of nonslip boundary conditions for the lattice Boltzmann BGK model, J. Stat. Phys. 87, 115-136 (1997). 
Table 1. The expressions for $\chi_{q}, \chi_{t}, \gamma_{q}$, and $\gamma_{t}$ in Schemes 1, 2 and 3 in Eqs. (15a, 15b) for general conjugate interface jump conditions in 2-D problems.

\begin{tabular}{|c|c|c|c|c|}
\hline & Scheme $1(0 \leq \Delta \leq 0.5)$ & Scheme $1(0.5<\Delta \leq 1)$ & Scheme $2(0 \leq \Delta \leq 1)$ & Scheme $3(0 \leq \Delta \leq 1)$ \\
\hline$\chi_{q}$ & $2(1-\Delta) / P$ & $1 / P$ & $(3-2 \Delta) /[(2 \Delta+1) P]$ & $(3-2 \Delta) /(2 P)$ \\
\hline$\chi_{t}$ & $\sigma\left((1-\Delta) \sin \theta+\frac{3-2 \Delta}{2} \cos \theta\right) /(3 P)$ & $\sigma\left(\frac{1}{2} \sin \theta+\frac{3-2 \Delta}{2} \cos \theta\right) /(3 P)$ & $\sigma\left[\frac{(3 / 2)(3-2 \Delta)}{(2 \Delta+1)} \sin \theta+\frac{2}{3-2 \Delta} \cos \theta\right] /(3 P)$ & $(3-2 \Delta) \sigma(\sin \theta+\cos \theta) /(6 P)$ \\
\hline$\gamma_{q}$ & $1 / P$ & $2 \Delta / P$ & $(2 \Delta+1) /[(3-2 \Delta) P]$ & $(2 \Delta+1) /(2 P)$ \\
\hline$\gamma_{t}$ & $-\left(\frac{1}{2} \sin \theta+\frac{2 \Delta+1}{2} \cos \theta\right) /(3 P)$ & $-\left(\Delta \sin \theta+\frac{2 \Delta+1}{2} \cos \theta\right) /(3 P)$ & $-\left[\frac{(3 / 2)(2 \Delta+1)}{(3-2 \Delta)} \sin \theta+\frac{2 \Delta+1}{2} \cos \theta\right] /(3 P)$ & $-(2 \Delta+1)(\sin \theta+\cos \theta) /(6 P)$ \\
\hline$P$ & $(1-\Delta)(\sigma+1) \sin \theta+$ & $\Delta(\sigma+1) \sin \theta+$ & $(3 / 2)(\sigma+1) \sin \theta+\left(\frac{2 \Delta+1}{2} \sigma+\frac{3-2 \Delta}{2}\right) \cos \theta$ & $(2 \Delta+1)(3-2 \Delta) / 4 \times$ \\
$((3-2 \Delta) \sigma / 2+(1-\Delta)(1+2 \Delta)] \cos \theta$ & {$[\Delta(3-2 \Delta) \sigma+(2 \Delta+1) / 2] \cos \theta$} & $(\sigma+1)(\sin \theta+\cos \theta)$ \\
\hline
\end{tabular}


Table 2. Coefficients in Schemes 1, 2 and 3 for the decoupled conjugate interface treatment in Eqs. (22a, 22b) for 2-D problems (Note: there is a typo in Tables I and II in [30] for $B_{2}^{s}$ in Scheme $\left.1(0 \leq \Delta \leq 0.5)\right)$.

\begin{tabular}{|c|c|c|c|c|}
\hline & Scheme $1(0 \leq \Delta \leq 0.5)$ & Scheme $1(0.5<\Delta \leq 1)$ & Scheme $2(0 \leq \Delta \leq 1)$ & Scheme $3(0 \leq \Delta \leq 1)$ \\
\hline$A_{1}^{f}$ & {$[-\Delta(3-2 \Delta) \sigma+(1-\Delta)(2 \Delta+1)] / P$} & $\left(-\frac{3-2 \Delta}{2} \sigma+\frac{2 \Delta+1}{2}\right) / P$ & {$\left[\frac{3-2 \Delta}{2}-(1-\Delta)(2 \Delta+1) \sigma\right] / P$} & $-(2 \Delta+1)(3-2 \Delta)(\sigma-1) /(4 P)$ \\
\hline$A_{2}^{f}$ & $(2 \Delta-1)\left[\frac{(3-2 \Delta)}{2} \sigma-(1-\Delta)\right] / P$ & $\left(\frac{1-2 \Delta}{2}\right) / P$ & $(1-2 \Delta)\left[\frac{3-2 \Delta}{2(2 \Delta+1)}-\frac{1-2 \Delta}{2} \sigma\right] / P$ & $(2 \Delta-1)(3-2 \Delta)(\sigma-1) /(4 P)$ \\
\hline$A_{3}^{f}$ & $(1-\Delta)(2 \Delta-1) / P$ & $(2 \Delta-1)\left(\frac{3-2 \Delta}{2} \sigma+\frac{1}{2}\right) / P$ & $(2 \Delta-1)\left[\frac{3-2 \Delta}{2(2 \Delta+1)}+\sigma\right] / P$ & $(2 \Delta-1)(3-2 \Delta)(\sigma+1) /(4 P)$ \\
\hline$B_{1}^{f}$ & $\frac{(3-2 \Delta)^{2}}{2} \sigma / P$ & $\frac{(3-2 \Delta)^{2}}{2} \sigma / P$ & $\frac{(3-2 \Delta)^{2}}{2} \sigma / P$ & $2(3-2 \Delta)^{2} \sigma /(4 P)$ \\
\hline$B_{2}^{f}$ & $(1-\Delta)(2 \Delta-1) \sigma / P$ & $(2-\Delta)(2 \Delta-1) \sigma / P$ & $\frac{\Delta(2 \Delta-1)(3-2 \Delta)}{2 \Delta+1} \sigma / P$ & $2(2 \Delta-1)(3-2 \Delta) \sigma /(4 P)$ \\
\hline$B_{3}^{f}$ & $\left(\frac{2 \Delta-1}{2}\right) \sigma / P$ & $\left(\frac{1-2 \Delta}{2}\right) \sigma / P$ & $\frac{(2 \Delta-1)(3-2 \Delta)}{2(2 \Delta+1)} \sigma / P$ & 0 \\
\hline$A_{1}^{s}$ & $\left(\frac{3-2 \Delta}{2} \sigma-\frac{2 \Delta+1}{2}\right) / P$ & {$[\Delta(3-2 \Delta) \sigma-(1-\Delta)(2 \Delta+1)] / P$} & {$\left[\frac{2 \Delta+1}{2} \sigma-\Delta(3-2 \Delta)\right] / P$} & $-(3-2 \Delta)(2 \Delta+1)(1-\sigma) /(4 P)$ \\
\hline$A_{2}^{s}$ & $\left(\frac{2 \Delta-1}{2}\right) \sigma / P$ & $(1-2 \Delta)\left(-\Delta \sigma+\frac{2 \Delta+1}{2}\right) / P$ & $(2 \Delta-1)\left[\frac{2 \Delta+1}{2(3-2 \Delta)} \sigma-\frac{2 \Delta-1}{2}\right] / P$ & $(1-2 \Delta)(2 \Delta+1)(1-\sigma) /(4 P)$ \\
\hline
\end{tabular}




\begin{tabular}{|c|c|c|c|c|}
\hline$A_{3}^{s}$ & $(1-2 \Delta)\left(\frac{\sigma}{2}+\frac{2 \Delta+1}{2}\right) / P$ & $\Delta(1-2 \Delta) \sigma / P$ & $(1-2 \Delta)\left[\frac{2 \Delta+1}{2(3-2 \Delta)} \sigma+1\right] / P$ & $(1-2 \Delta)(2 \Delta+1)(1+\sigma) /(4 P)$ \\
\hline$B_{1}^{s}$ & $\frac{(2 \Delta+1)^{2}}{2} / P$ & $\frac{(2 \Delta+1)^{2}}{2} / P$ & $\frac{(2 \Delta+1)^{2}}{2} / P$ & $2(2 \Delta+1)^{2} /(4 P)$ \\
\hline$B_{2}^{s}$ & $(1+\Delta)(1-2 \Delta) / P$ & $\Delta(1-2 \Delta) / P$ & $\frac{(1-\Delta)(1-2 \Delta)(2 \Delta+1)}{3-2 \Delta} / P$ & $2(1-2 \Delta)(2 \Delta+1) /(4 P)$ \\
\hline$B_{3}^{s}$ & $\left(\frac{2 \Delta-1}{2}\right) / P$ & $\left(\frac{1-2 \Delta}{2}\right) / P$ & $(3-2 \Delta) /[(2 \Delta+1) P]$ & $(3-2 \Delta) /(2 P)$ \\
\hline$\chi_{q}$ & $2(1-\Delta) / P$ & $1 / P$ & $(3-2 \Delta) \sigma /(6 P)$ & $(3-2 \Delta) \sigma /(6 P)$ \\
\hline$\chi_{t}$ & $(3-2 \Delta) \sigma /(6 P)$ & $(3-2 \Delta) \sigma /(6 P)$ & $(2 \Delta+1) /[(3-2 \Delta) P]$ & $(2 \Delta+1) /(2 P)$ \\
\hline$\gamma_{q}$ & $1 / P$ & $2 \Delta / P$ & $-(2 \Delta+1) /(6 P)$ & $-(2 \Delta+1) /(6 P)$ \\
\hline$\gamma_{t}$ & $-(2 \Delta+1) /(6 P)$ & $-(2 \Delta+1) /(6 P)$ & $\frac{2 \Delta+1}{2} \sigma+\frac{3-2 \Delta}{2}$ & $(2 \Delta+1)(3-2 \Delta)(\sigma+1) / 4$ \\
\hline$P$ & $(3-2 \Delta) \sigma / 2+(1-\Delta)(1+2 \Delta)$ & $\Delta(3-2 \Delta) \sigma+(2 \Delta+1) / 2$ & $(3-1)$ \\
\hline
\end{tabular}

(Table 2 continued) 


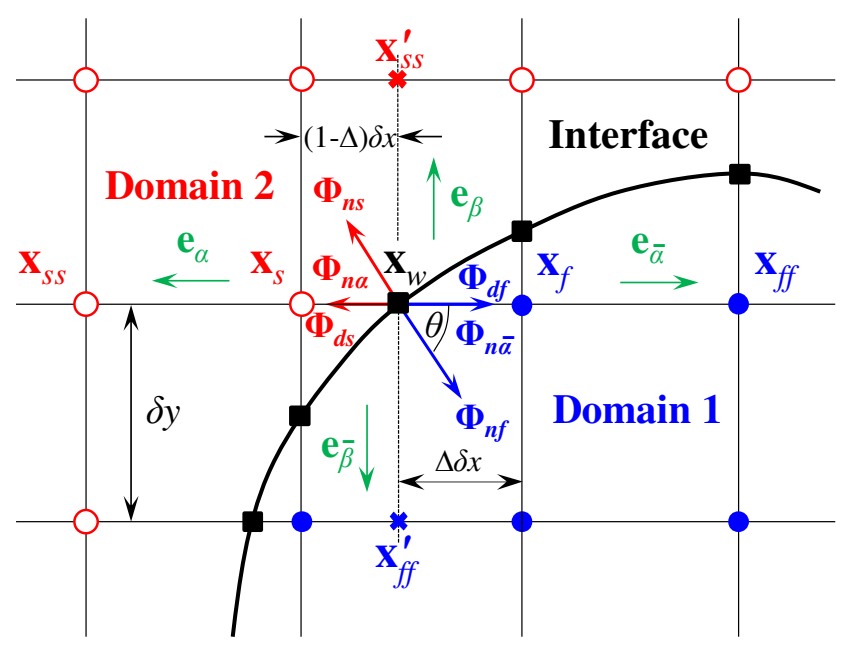

Fig. 1. Illustration of a curved interface in the square lattice with a temperature (concentration) jump condition $\Phi_{d f}=\Phi_{d s}+\phi_{\text {jump }}$, and a flux jump condition $\Phi_{n f}=-\sigma \Phi_{n s}+q_{\text {jump }}$ (see the main text for notations; solid circles: lattice nodes in Domain 1, solid squares: interface nodes, open circles: lattice nodes in Domain 2).

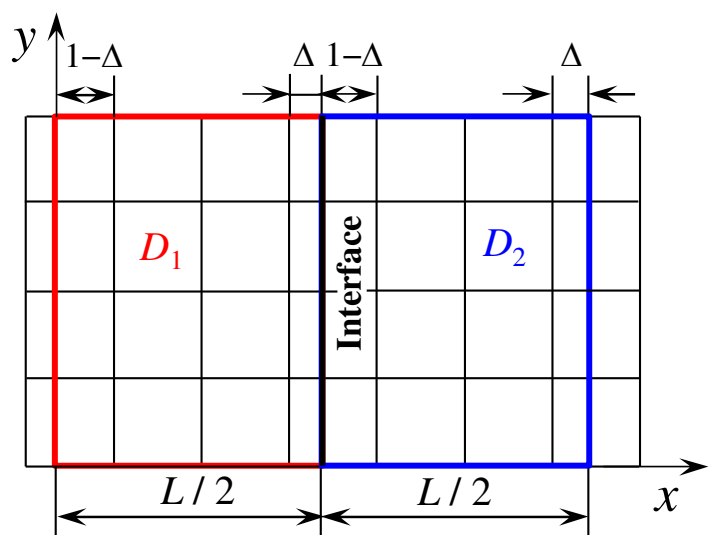

Fig. 2. Schematic depiction of the computational domain for the 1D steady diffusion problem with jump conditions at the interface. 


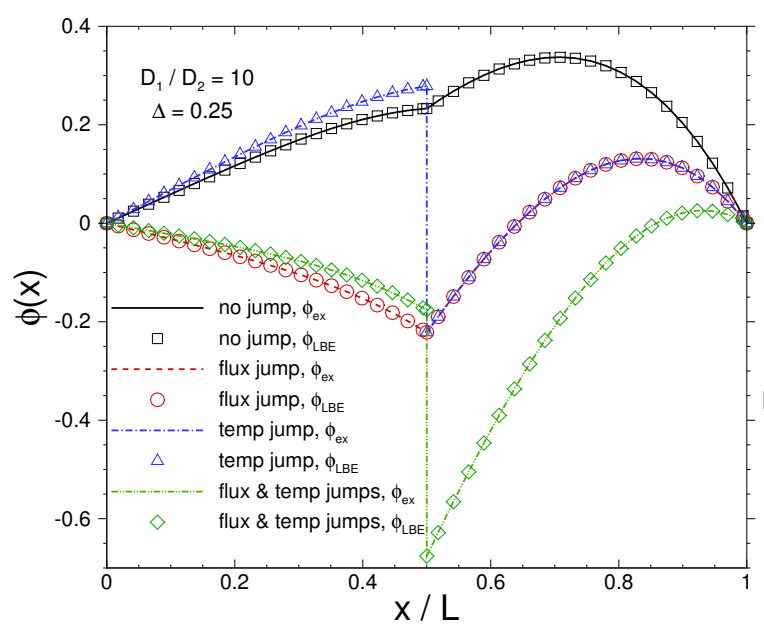

(a)

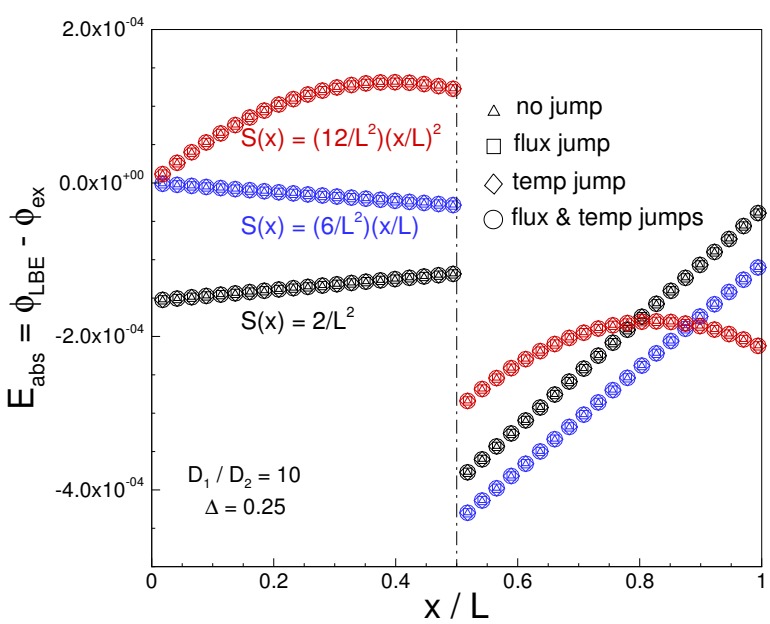

(b)

Fig. 3. (a) Comparison of exact and LBE results for the profiles of $\phi$ with various interfacial jump conditions and a quadratic heat source, and (b) the absolute errors $E_{\mathrm{abs}}=\phi_{\mathrm{LBE}}-\phi_{\mathrm{ex}}$ with various jump conditions and heat sources.

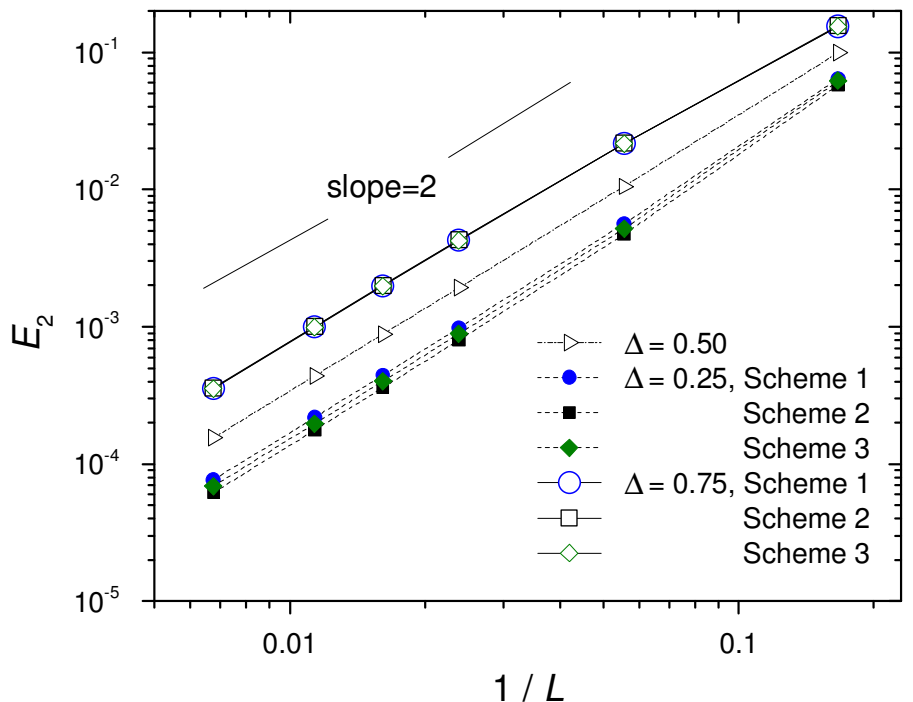

Fig. 4. Relative $L_{2}$-norm error, $E_{2}$, for the interior field versus the grid resolution, $1 / L$, for the $1 \mathrm{D}$ diffusion problem with $\phi_{\text {jump }}=0.5$ and $q_{\text {jump }}=-D_{1} / L$ at the interface and a quadratically distributed heat source. 


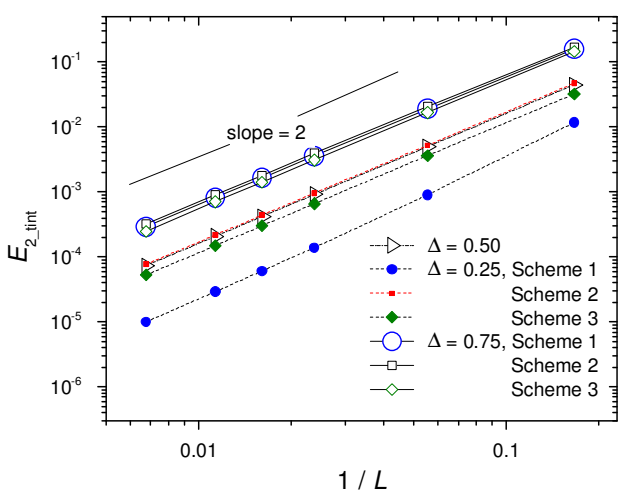

(a)

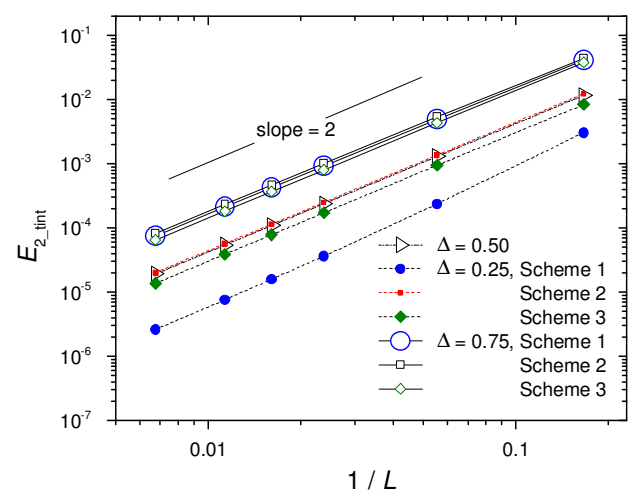

(b)

Fig. 5. Relative $L_{2}$-norm error, $E_{2 \_ \text {tint }}$, for the interfacial $\phi_{\text {int }}$ values: (a) $E_{2 \_ \text {tint_1 }}$ computed within Domain 1, and (b) $E_{2 \_ \text {tint_2 }}$ computed within Domain 2, versus $1 / L$ for the 1 D diffusion problem with $\phi_{\text {jump }}=0.5$ and $q_{\text {jump }}=-D_{1} / L$ at the interface and a quadratically distributed heat source.

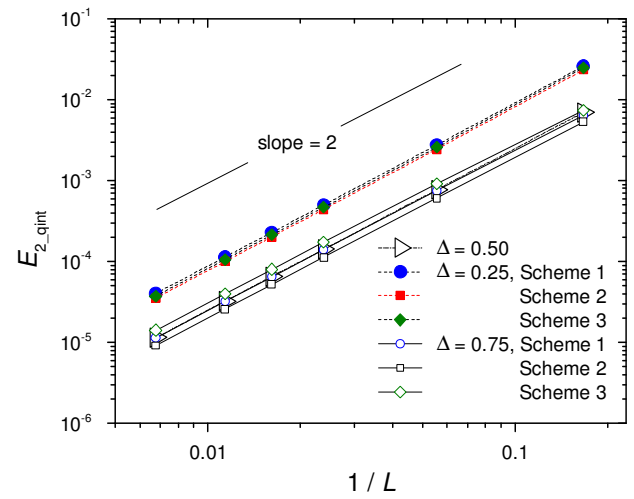

(a)

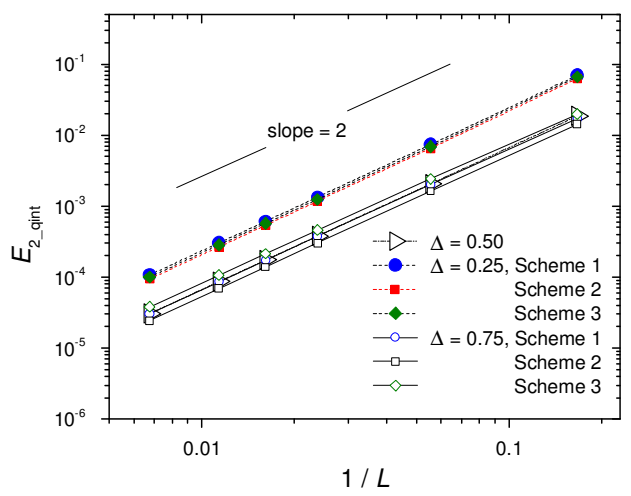

(b)

Fig. 6. Relative $L_{2}$-norm error, $E_{2 \_ \text {qint }}$, for the interfacial fluxes: (a) $E_{2 \_ \text {qint_1 }}$ computed within Domain 1, and (b) $E_{2 \_q i n t \_2}$ computed within Domain 2, versus $1 / L$ for the $1 \mathrm{D}$ diffusion problem with $\phi_{\text {jump }}=0.5$ and $q_{\text {jump }}=-D_{1} / L$ at the interface and a quadratically distributed heat source. 


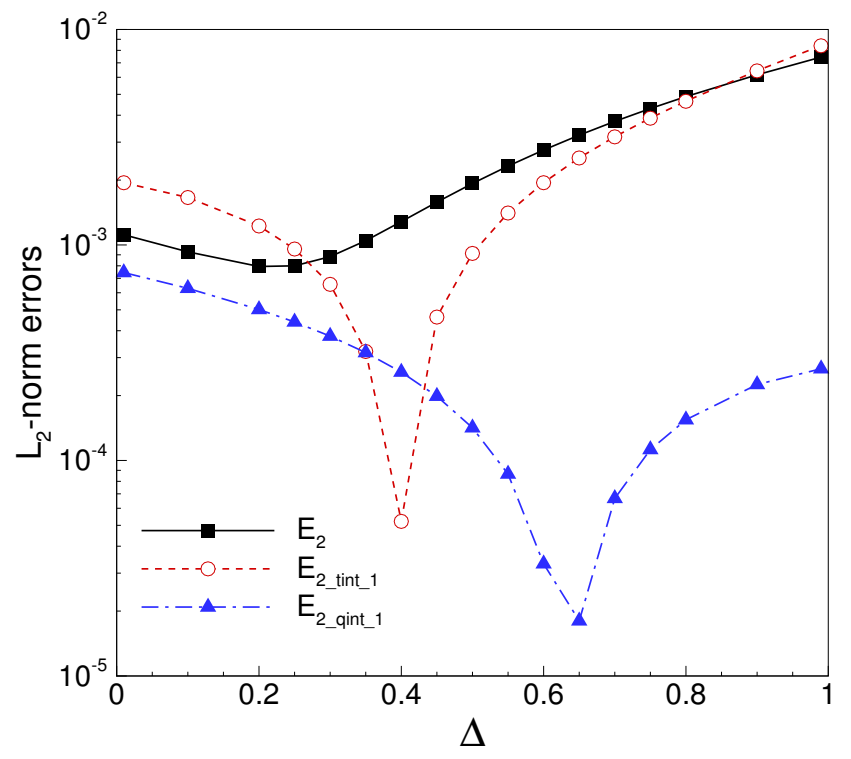

Fig. 7. Variations of the $L_{2}$-norm errors with the lattice link fraction $\Delta$ for the $1 \mathrm{D}$ diffusion problem with $\phi_{\text {jump }}=0.5$ and $q_{\text {jump }}=-D_{1} / L(L=42)$ at the interface and a quadratically distributed heat source.

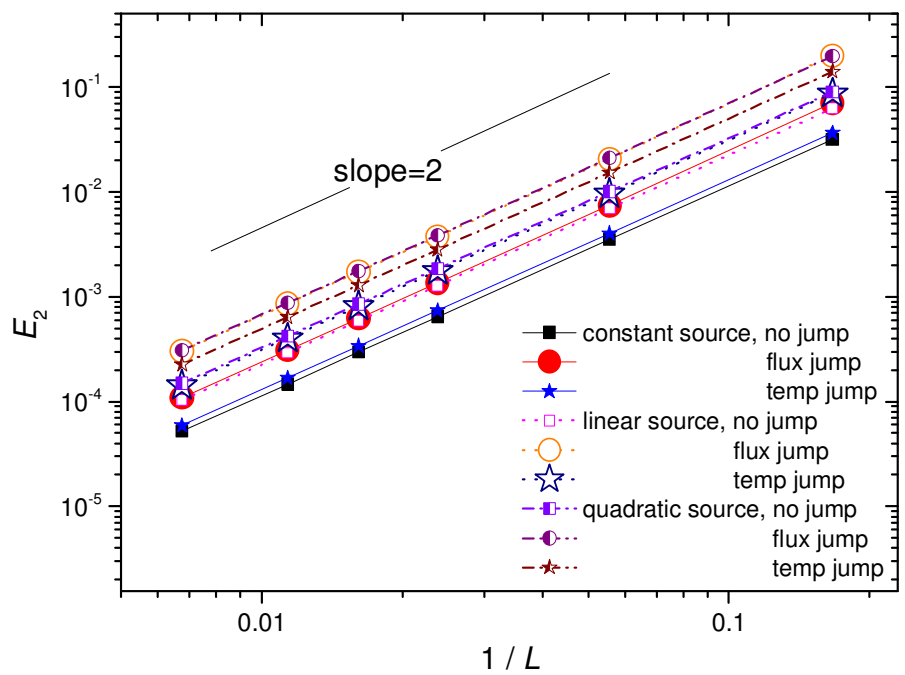

Fig. 8. $E_{2}$ for the interior field versus $1 / L$ for the $1 \mathrm{D}$ diffusion problem with different interfacial jump conditions and heat source distributions. 


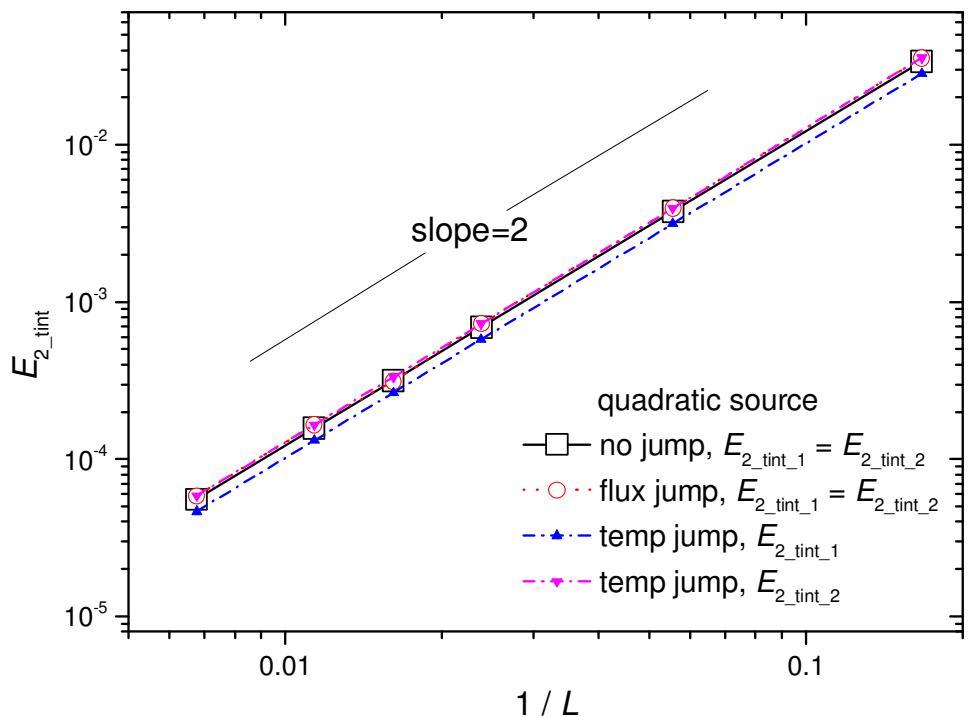

Fig. 9. $E_{2 \_ \text {tint }}$ for the interfacial $\phi_{\text {int }}$ values versus $1 / L$ for the $1 \mathrm{D}$ diffusion problem with different interfacial jump conditions and a quadratically distributed heat source.

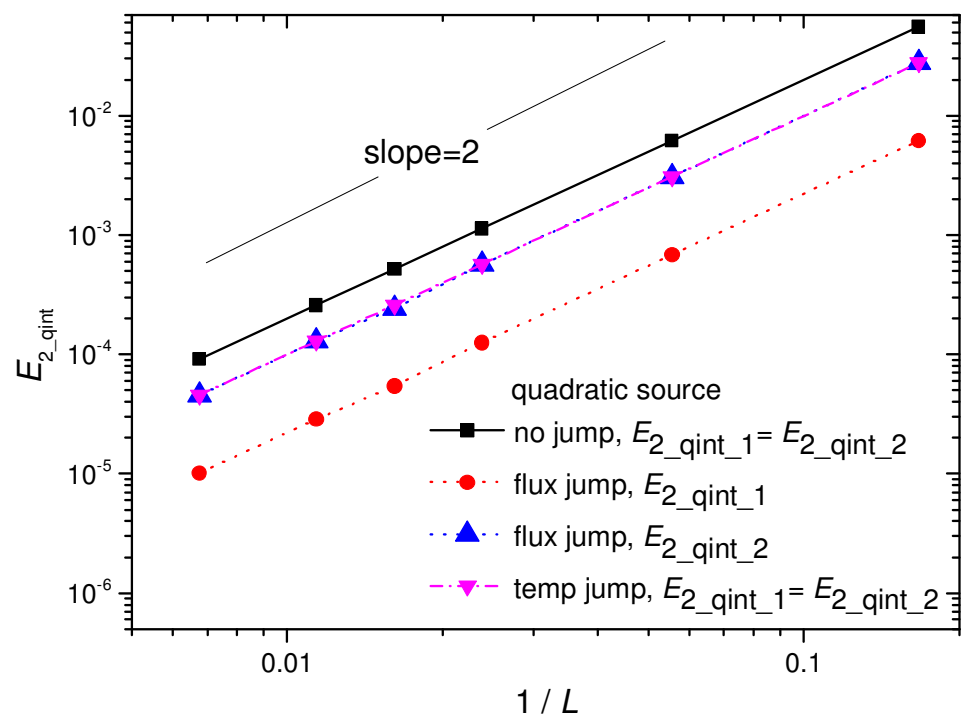

Fig. 10. $E_{2 \_q i n t}$ for the interfacial fluxes versus $1 / L$ for the $1 \mathrm{D}$ diffusion problem with different interfacial jump conditions and a quadratically distributed heat source. 


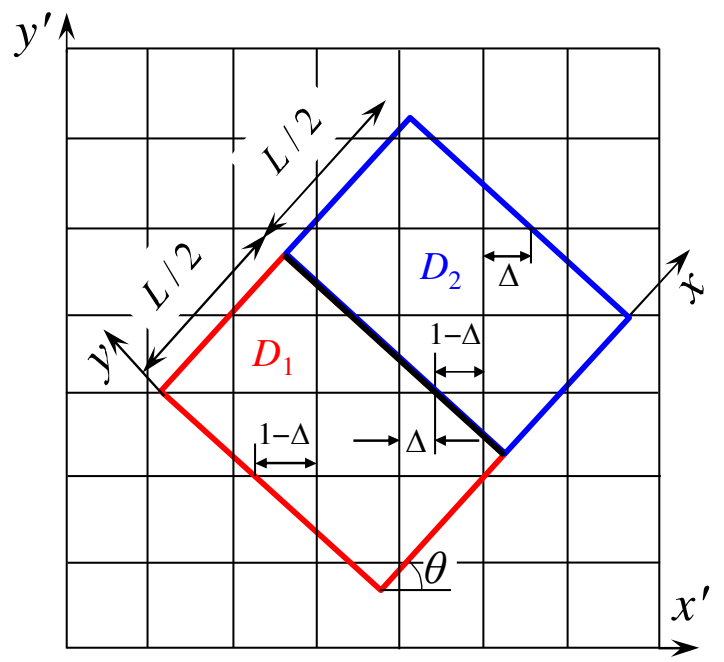

Fig. 11. Schematic depiction of the placement of the inclined two-solid slab in the square lattice.

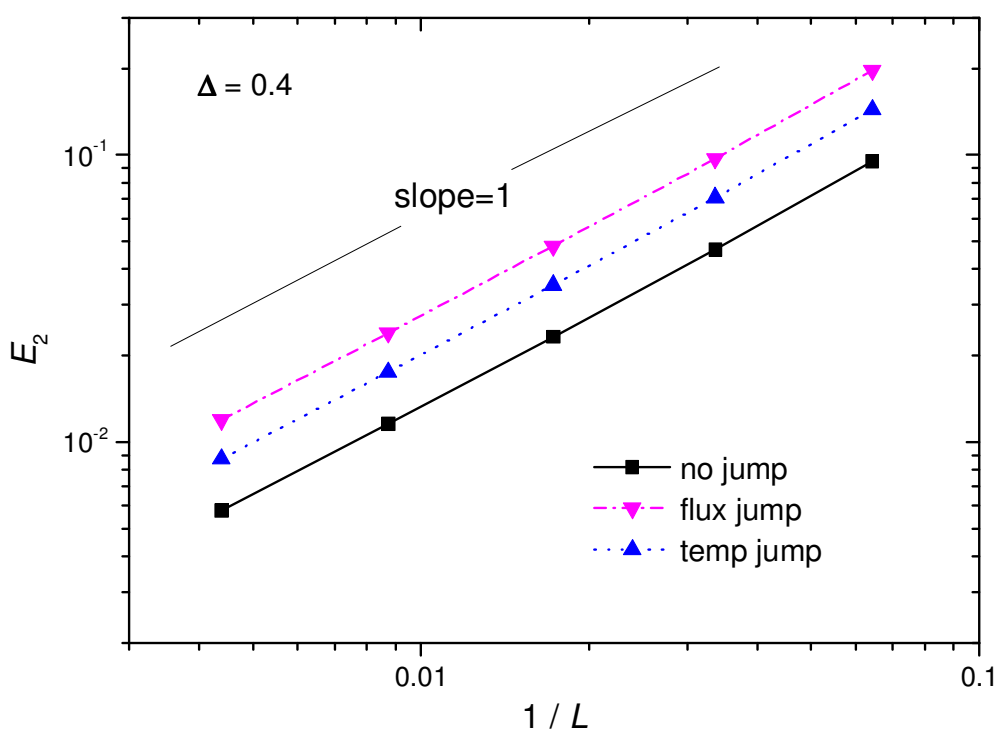

Fig. 12. $E_{2}$ for the interior field versus $1 / L$ for the $1 \mathrm{D}$ diffusion problem with different jump conditions at the inclined interface. 


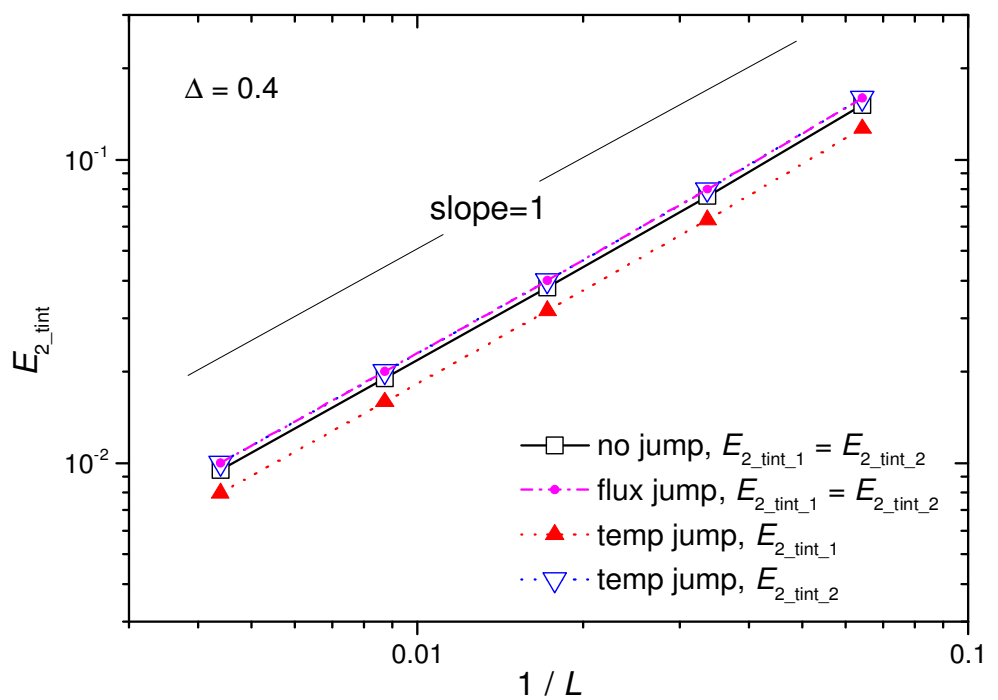

Fig. 13. $E_{2 \_ \text {tint }}$ for the interfacial $\phi_{\text {int }}$ values versus $1 / L$ for the $1 \mathrm{D}$ diffusion problem with different jump conditions at the inclined interface.

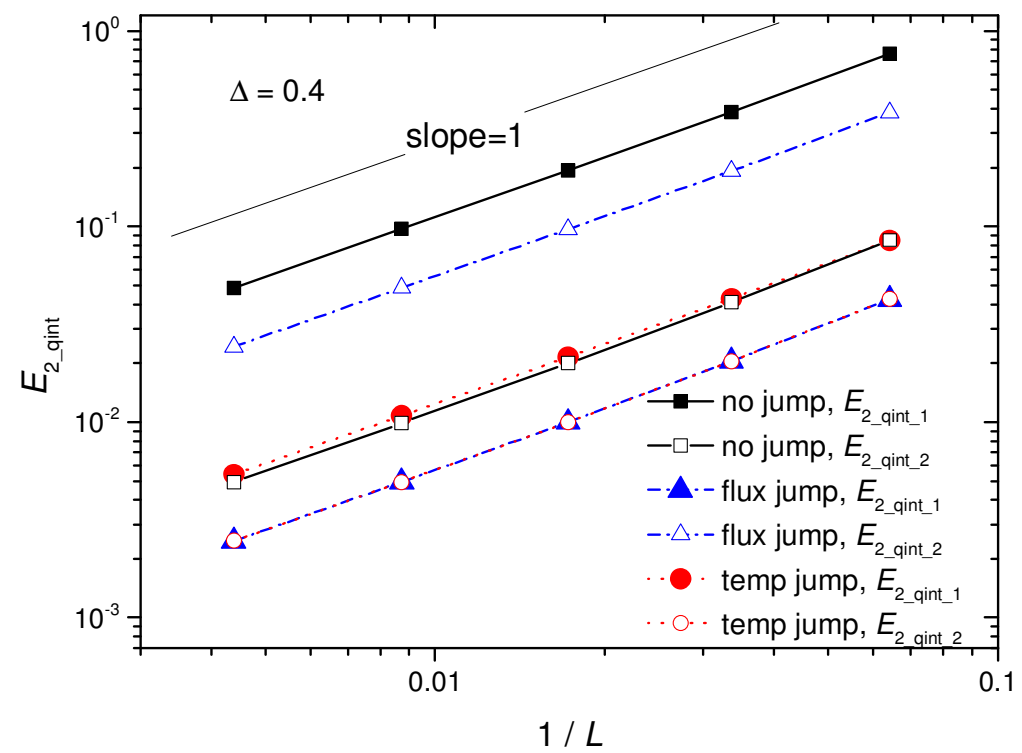

Fig. 14. $E_{2}$ qint for the interfacial fluxes versus $1 / L$ for the $1 \mathrm{D}$ diffusion problem with different jump conditions at the inclined interface. 


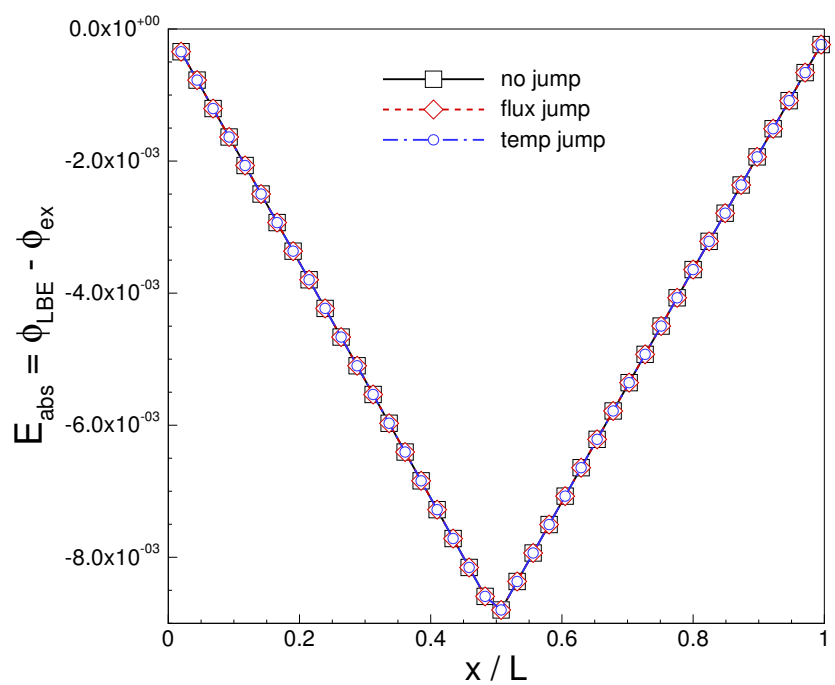

Fig. 15. Distributions of the absolute error $E_{\mathrm{abs}}=\phi_{\mathrm{LBE}}-\phi_{\mathrm{ex}}$ for the 1D diffusion problem with different jump conditions at the inclined interface.

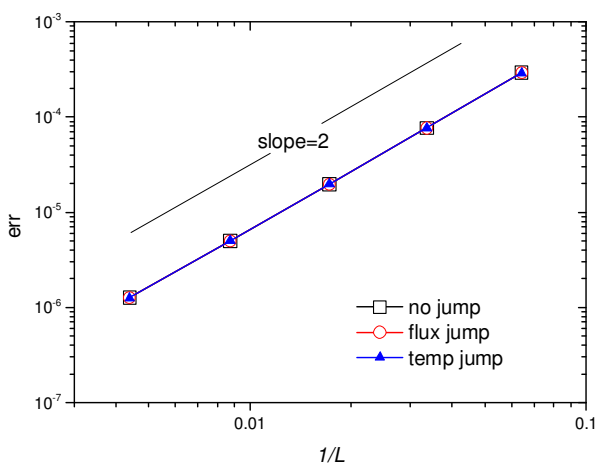

(a)

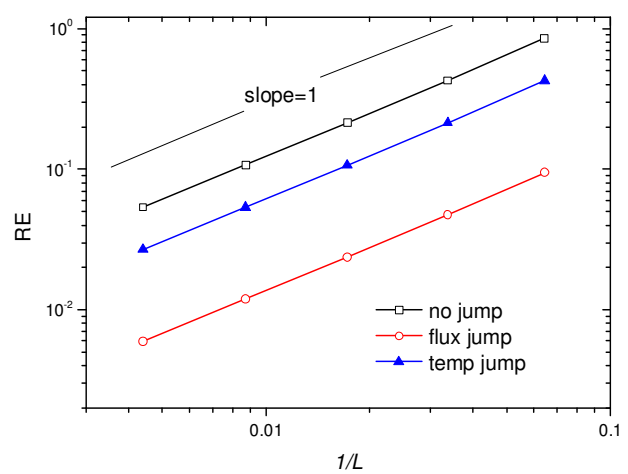

(b)

Fig. 16. (a) The absolute error, err $=\left|-D_{1} \frac{\partial \phi_{1}}{\partial x^{\prime}}\right|_{\mathrm{LBE}}+\left.D_{2} \frac{\partial \phi_{2}}{\partial x^{\prime}}\right|_{\mathrm{LBE}}-q_{\mathrm{jump}} \cos \theta \mid$, representing the satisfaction of the continuity or jump condition of the interfacial fluxes, and (b) the relative error, R.E. $=\operatorname{err} /\left|D_{1} \frac{\partial \phi_{1}}{\partial x^{\prime}}\right|_{\mathrm{ex}} \mid$, versus $1 / L$ for the $1 \mathrm{D}$ diffusion problem with different jump conditions at the inclined interface. 


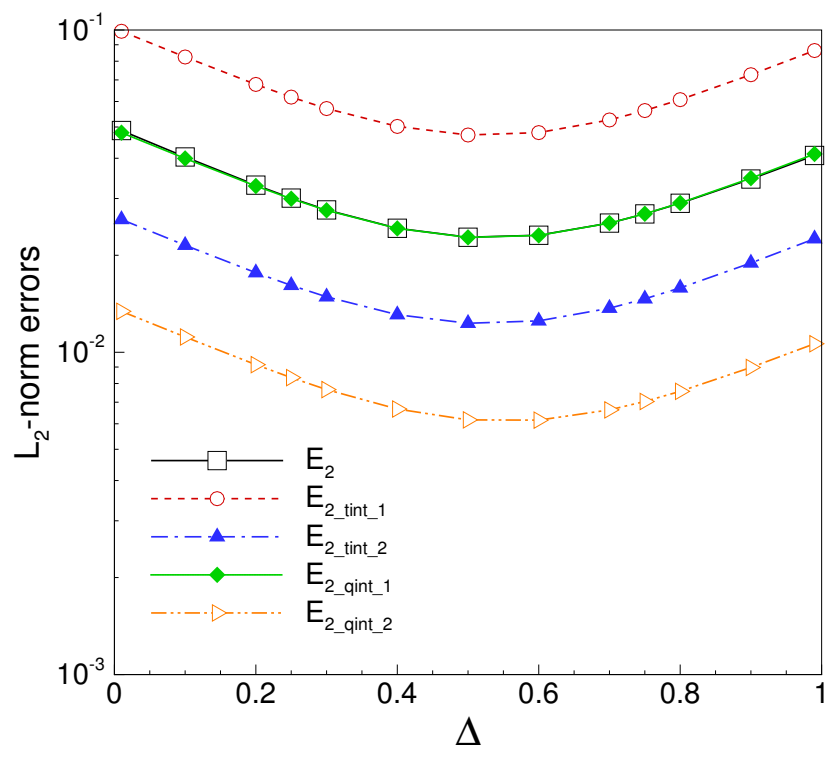

Fig. 17. Variations of the $L_{2}$-norm errors with the lattice link fraction $\Delta$ for the $1 \mathrm{D}$ diffusion problem with $\phi_{\text {jump }}=0.5$ and $q_{\text {jump }}=-D_{1} / L(L=41 / \cos (\pi / 4))$ at the inclined interface.

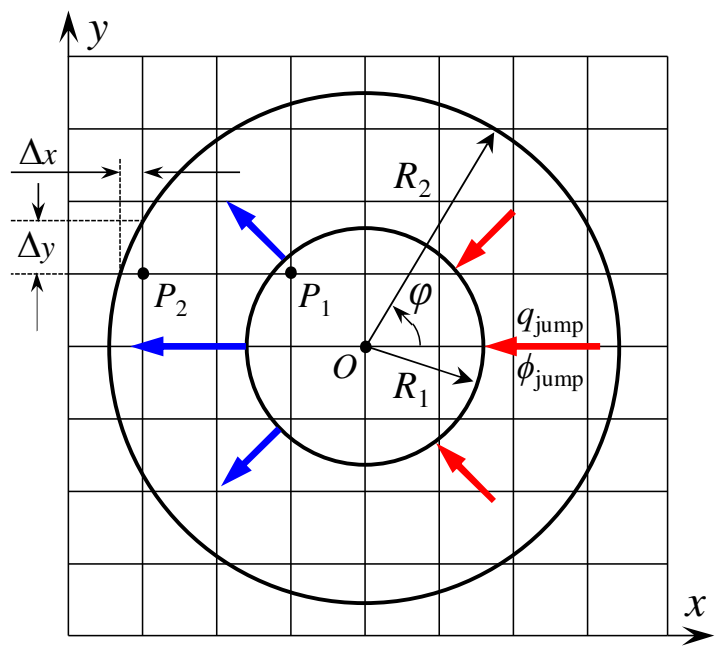

Fig. 18. Lattice layout and schematic depiction of the computational domain for diffusion in a 2D circular domain of two solid materials. 


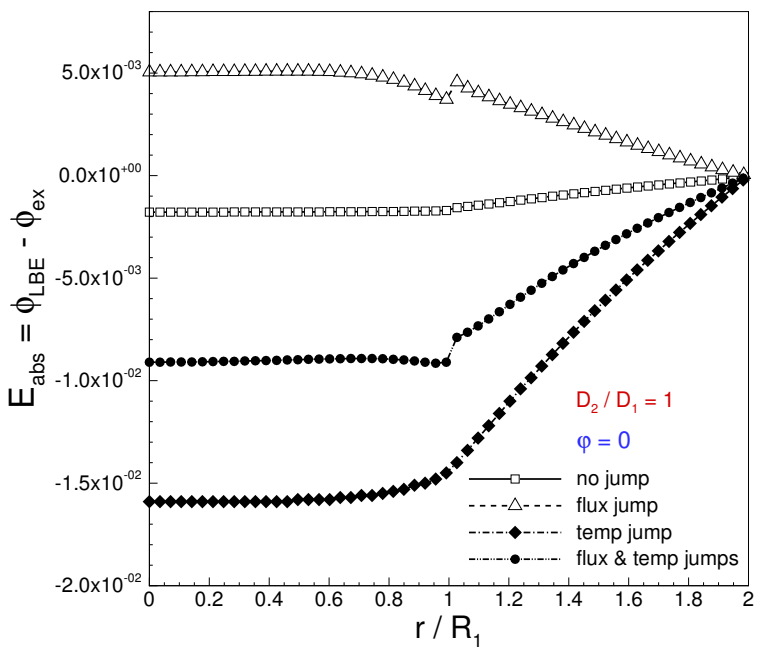

(a)

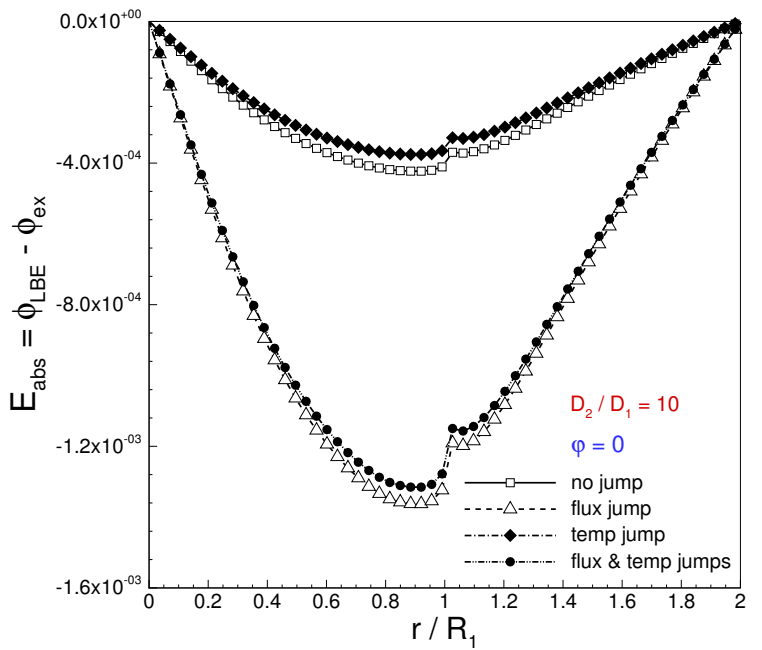

(c)

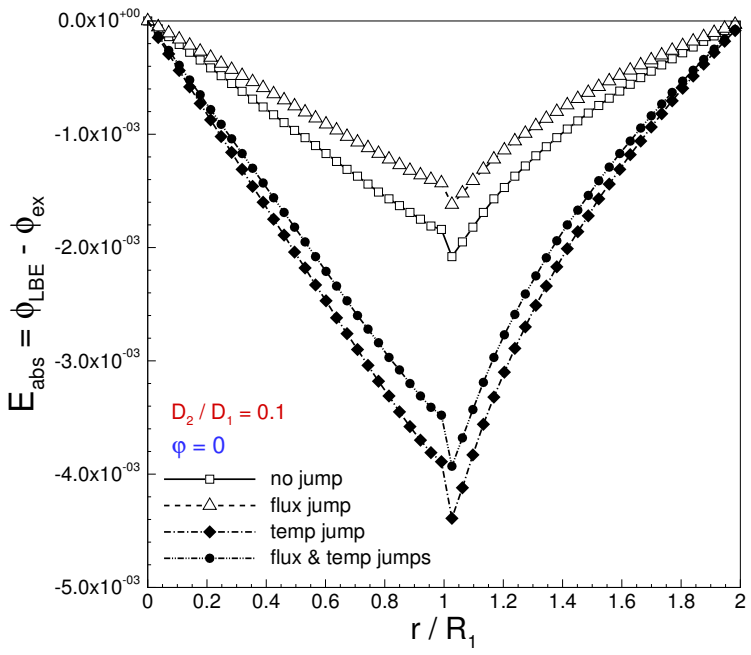

(e)

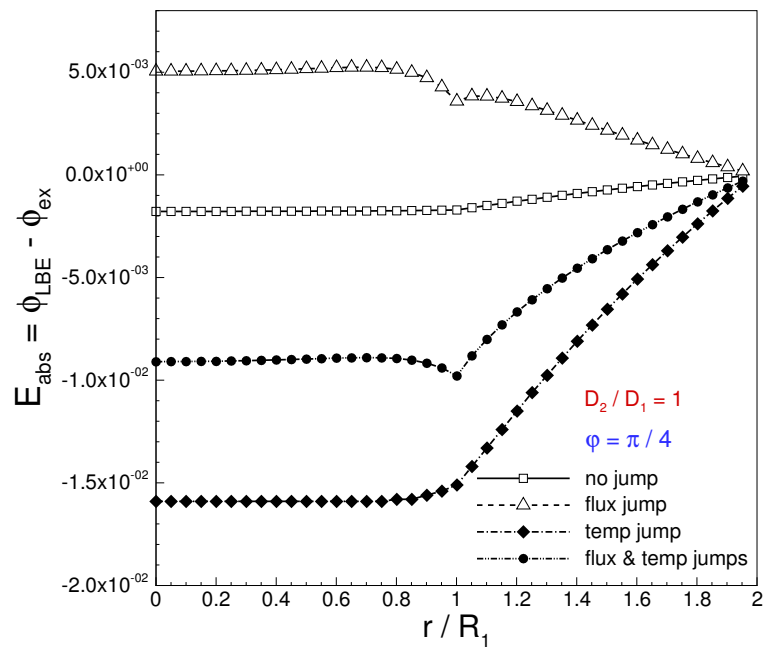

(b)

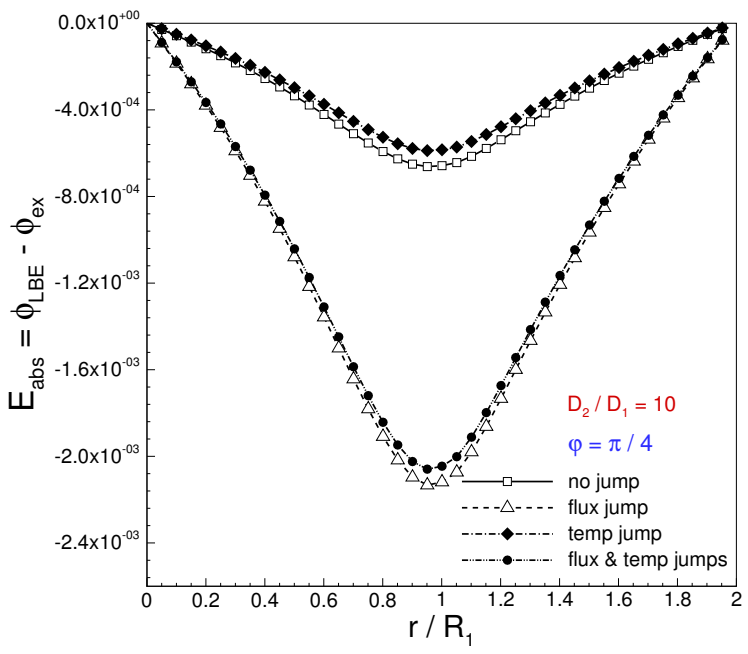

(d)

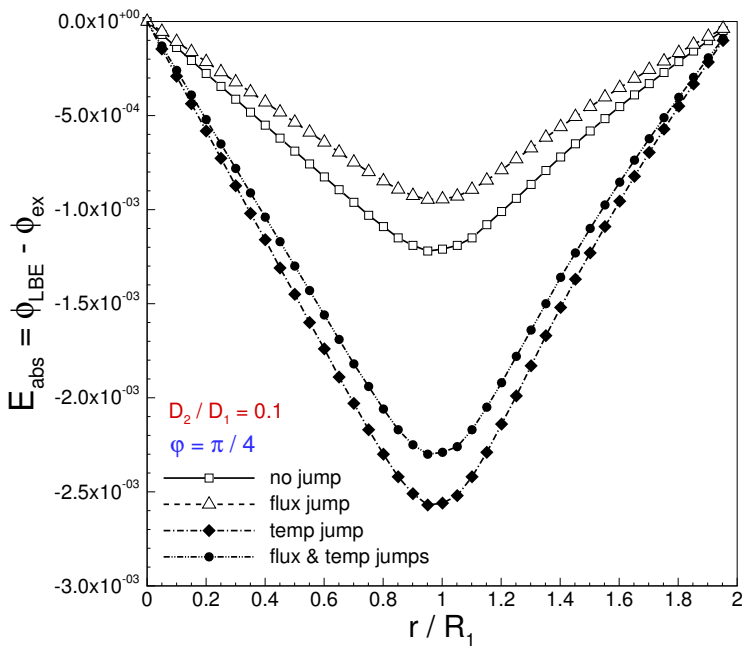

(f) 
Fig. 19. Comparison of the absolute errors $E_{\mathrm{abs}}=\phi_{\mathrm{LBE}}-\phi_{\mathrm{ex}}$ along the radius at constant $\varphi$ values for steady diffusion in a circle with various jump conditions: (a) $D_{2} / D_{1}=1, \varphi=0$, (b) $D_{2} / D_{1}=1, \varphi=\pi / 4$, (c) $D_{2} / D_{1}=10, \varphi=0$, (d) $D_{2} / D_{1}=10, \varphi=\pi / 4$, (e) $D_{2} / D_{1}=0.1, \varphi=0$, and (f) $D_{2} / D_{1}=0.1, \varphi=\pi / 4$.

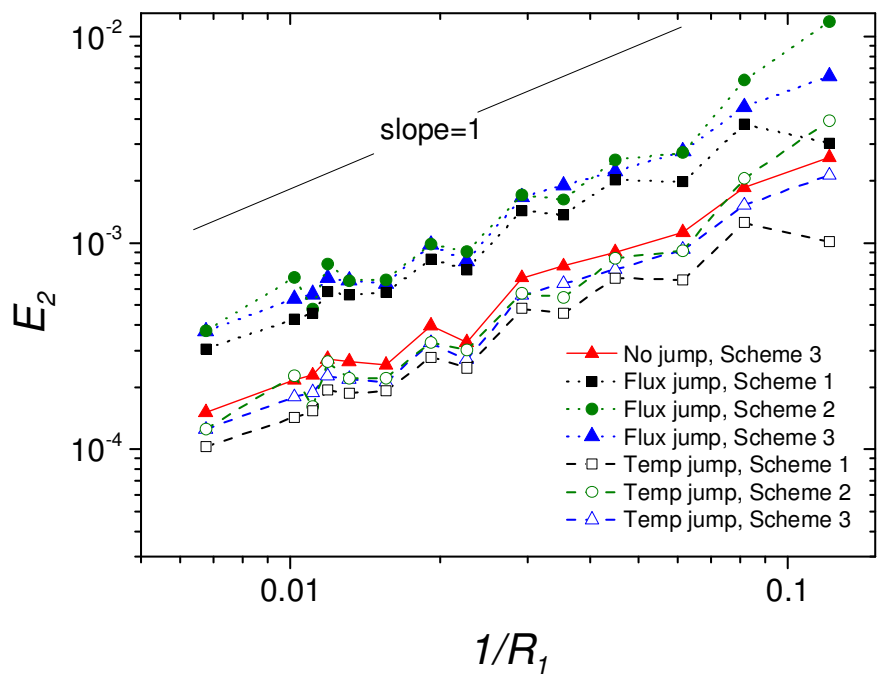

Fig. 20. $E_{2}$ for the interior field versus the grid resolution, $1 / R_{1}$, for the $2 \mathrm{D}$ diffusion problem in a circular domain with various jump conditions at the circular interface.

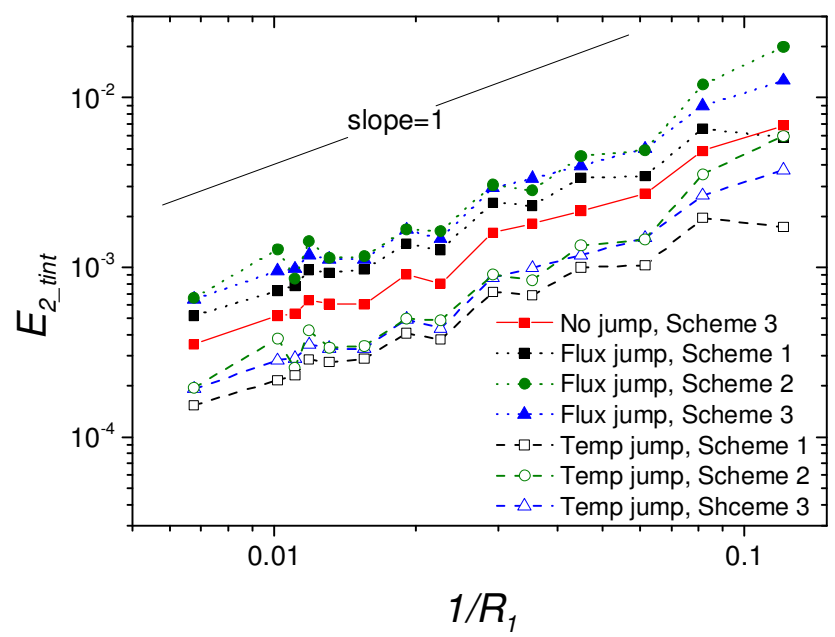

Fig. 21. $E_{2 \_ \text {tint }}$ for the interfacial $\phi_{\text {int }}$ values computed within Domain $1\left(r \leq R_{1}\right)$ versus $1 / R_{1}$ for the $2 \mathrm{D}$ diffusion problem in a circular domain with various jump conditions at the circular interface. 


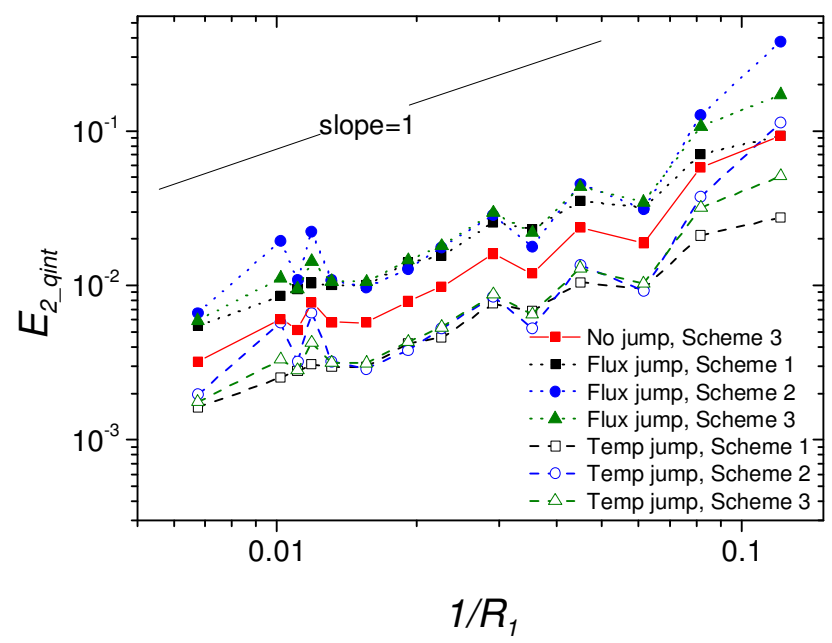

Fig. 22. $E_{2}$ qint for the interfacial fluxes computed within Domain $1\left(r \leq R_{1}\right)$ versus $1 / R_{1}$ for the $2 \mathrm{D}$ diffusion problem in a circular domain with various jump conditions at the circular interface.

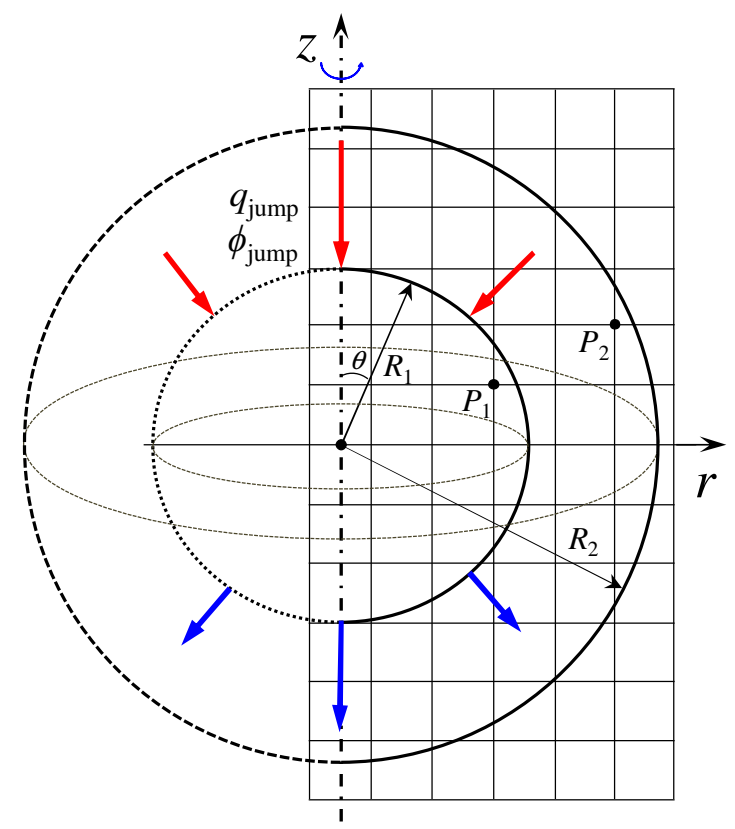

Fig. 23. Lattice layout and schematic depiction of the 2D axisymmetric computational domain for diffusion in a spherical domain. 


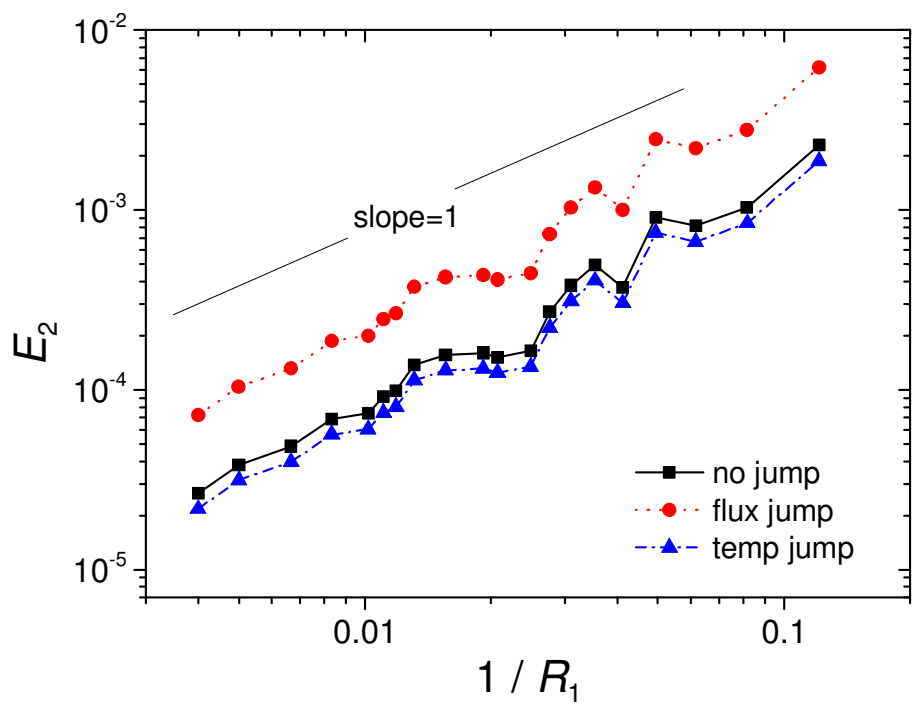

Fig. 24. $E_{2}$ for the interior field versus the grid resolution, $1 / R_{1}$, for diffusion in the spherical domain with various jump conditions at the circular interface.

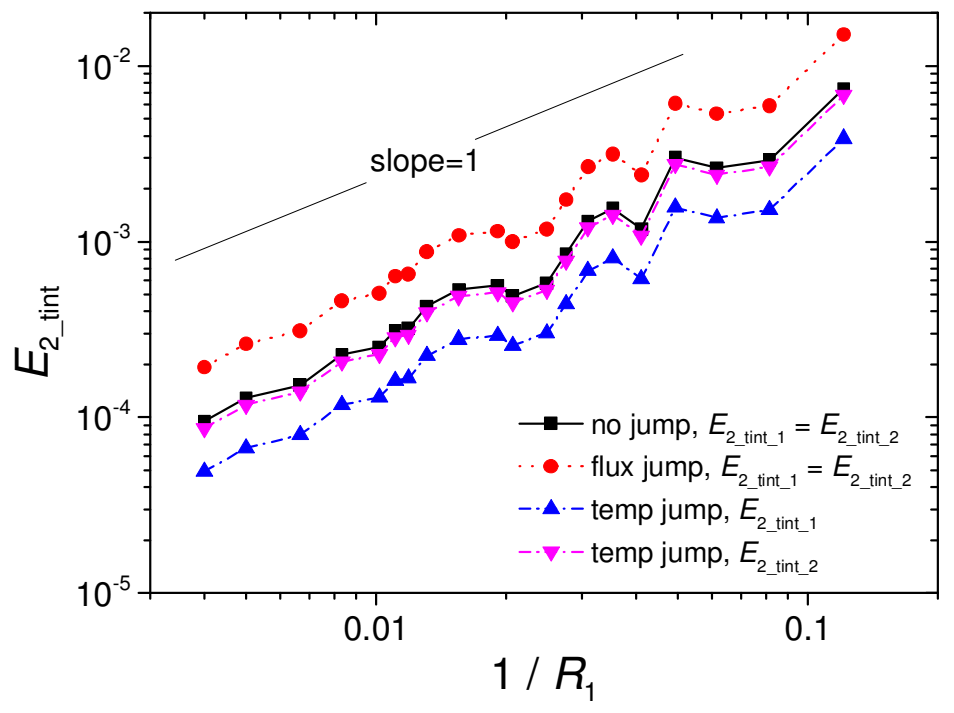

Fig. 25. $E_{2 \_ \text {tint }}$ for the interfacial $\phi_{\text {int }}$ values versus $1 / R_{1}$ for diffusion in the spherical domain with various jump conditions at the circular interface. 


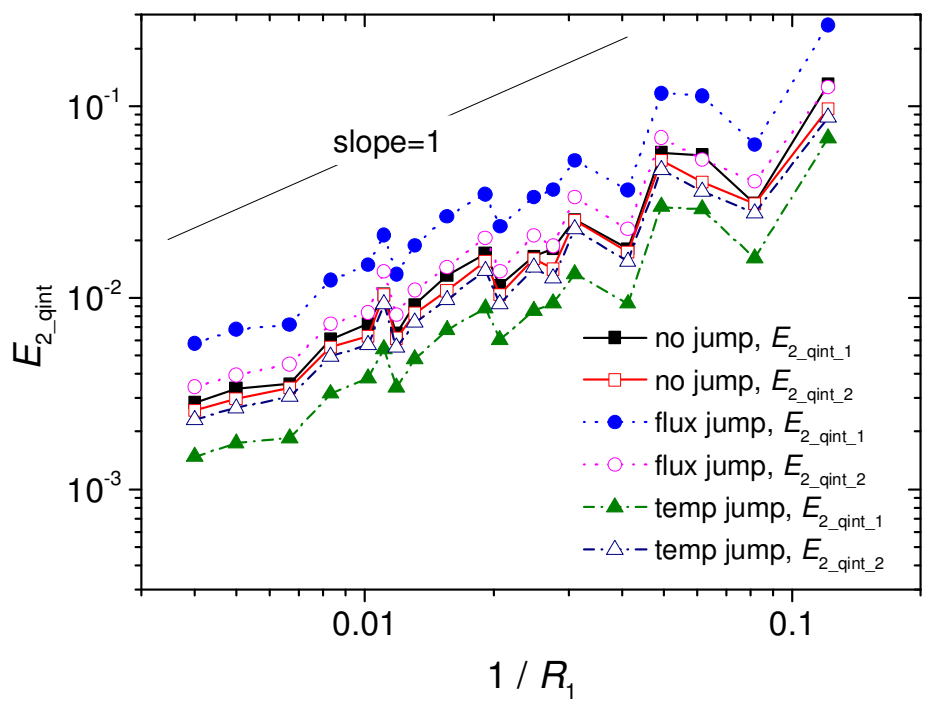

Fig. 26. $E_{2 \_q i n t}$ for the interfacial fluxes versus $1 / R_{1}$ for diffusion in the spherical domain with various jump conditions at the circular interface.

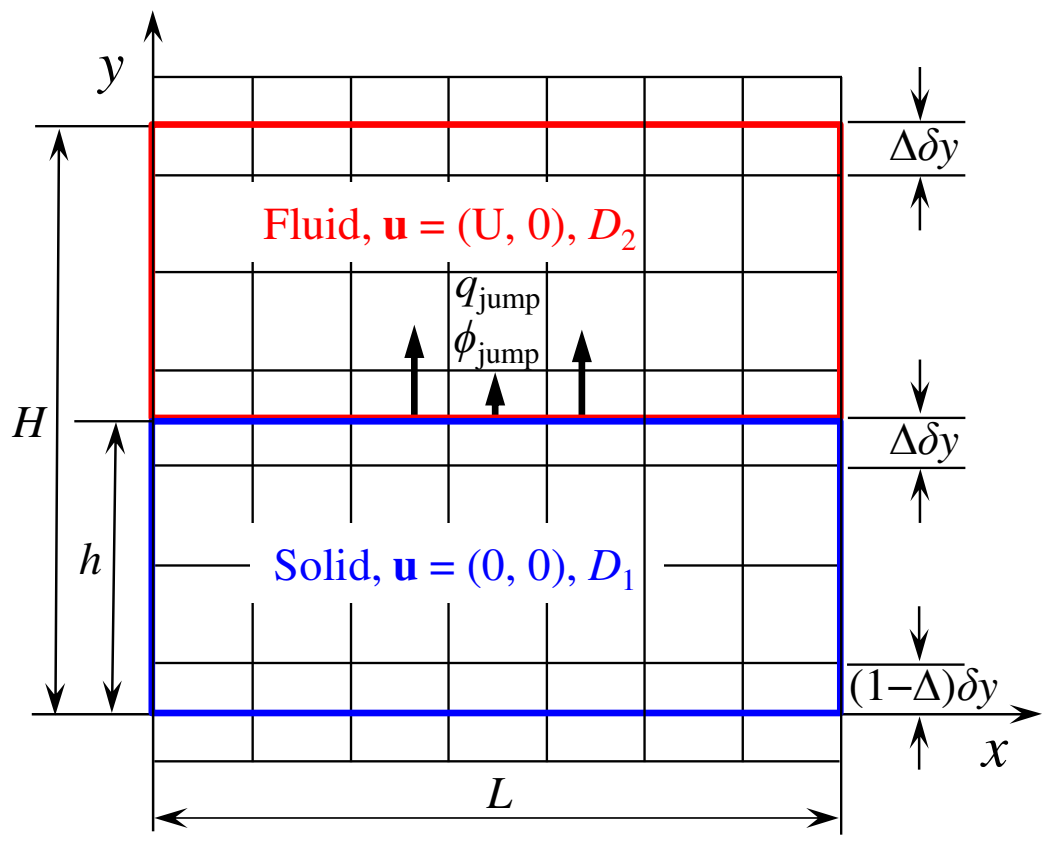

Fig. 27. Schematic depiction of the computational domain for the $2 \mathrm{D}$ convection-diffusion problem with jump conditions at the interface. 


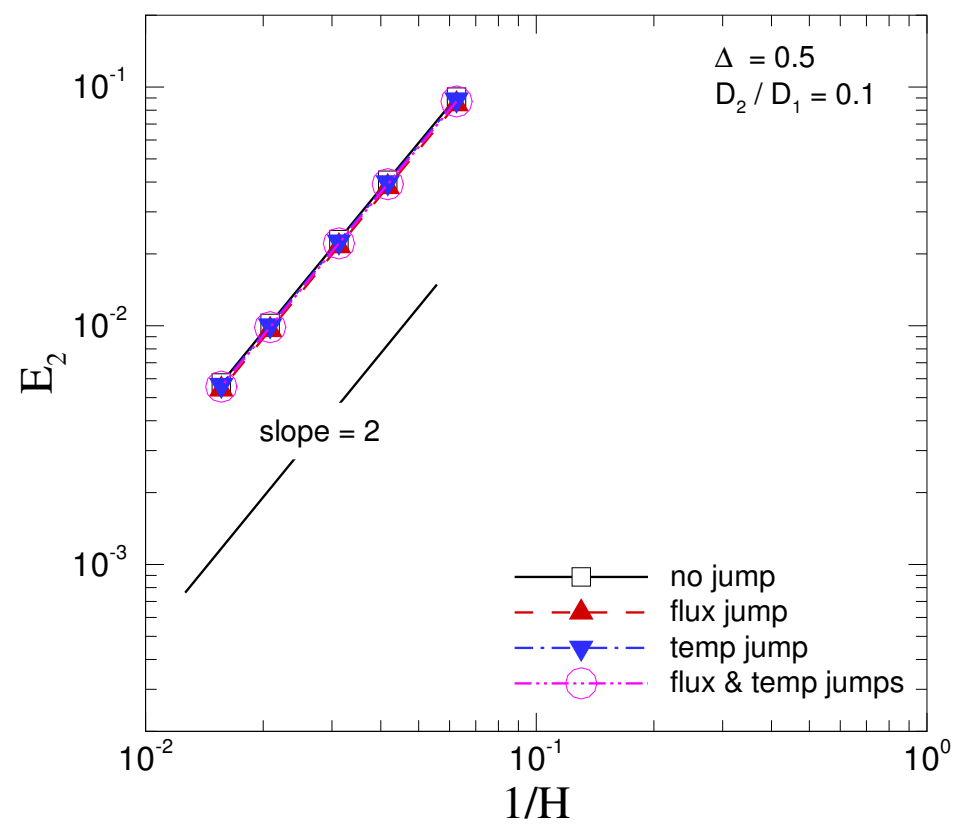

Fig. 28. $E_{2}$ for the interior field versus $1 / H$ for the $2 \mathrm{D}$ convection-diffusion problem with different jump conditions at the interface.

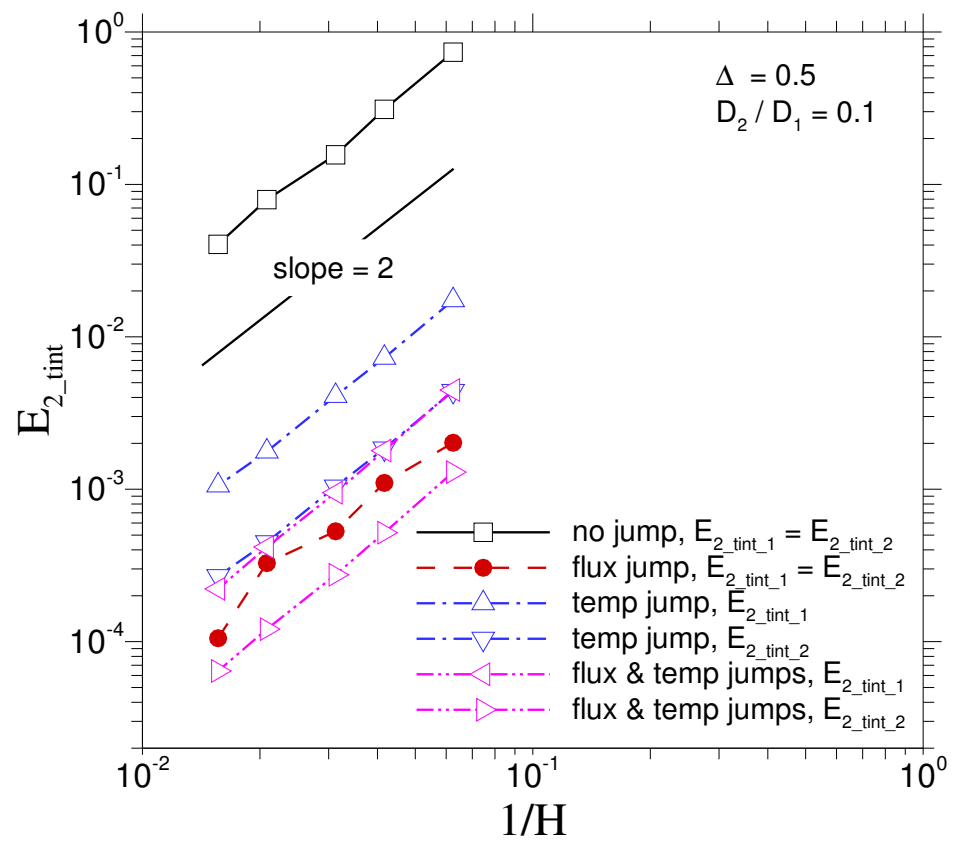

Fig. 29. $E_{2 \_ \text {tint }}$ for the interfacial $\phi_{\text {int }}$ values versus $1 / H$ for the $2 \mathrm{D}$ convection-diffusion problem with different jump conditions at the interface. 


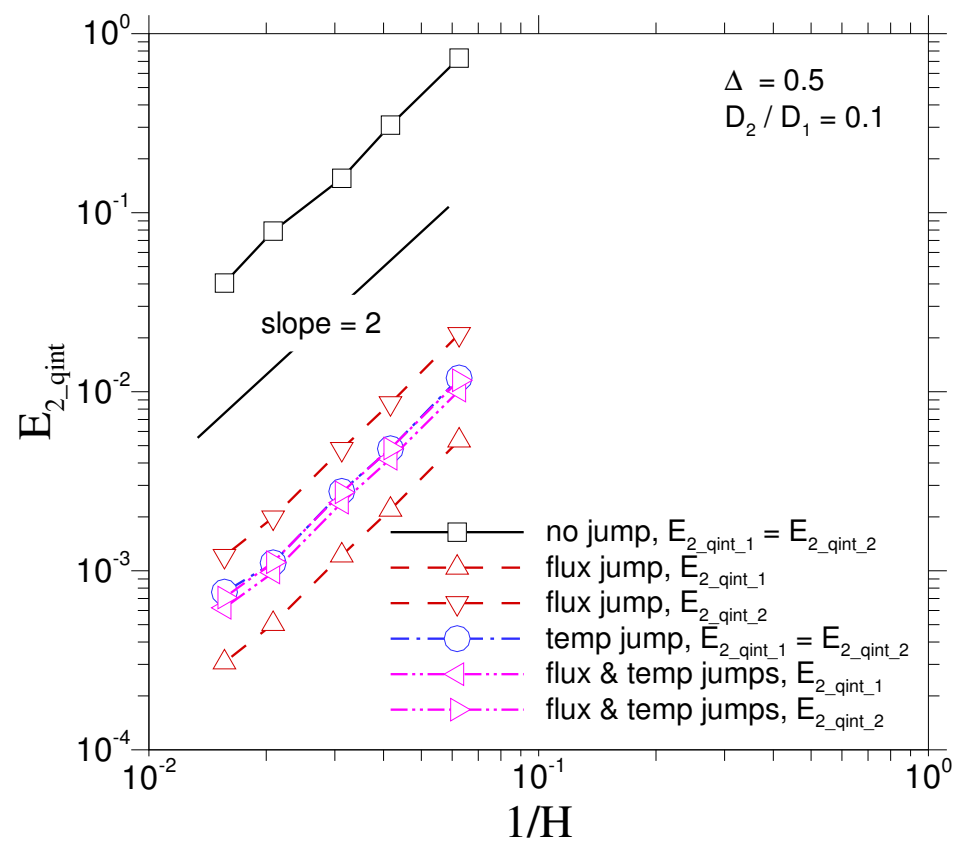

Fig. 30. $E_{2 \_ \text {qint }}$ for the interfacial fluxes versus $1 / H$ for the $2 \mathrm{D}$ convection-diffusion problem with different jump conditions at the interface.

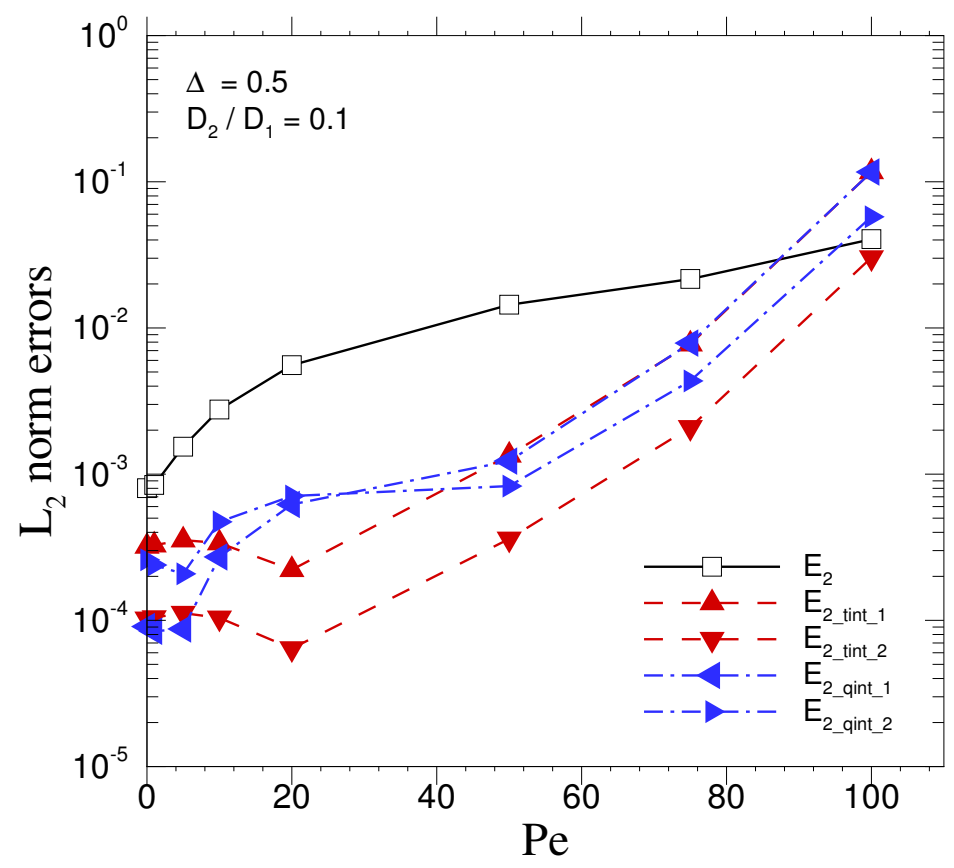

Fig. 31. $L_{2}$-norm errors versus the Péclet number for the $2 \mathrm{D}$ convection-diffusion problem with two jump conditions $\phi_{0}=0.5, q_{0}=D_{2} / h$ at the interface. 